- Supplementary material -

\title{
Evaluation of adding an olefin to mixtures of primary reference fuels and toluene to model the oxidation of a fully-blended gasoline
}

by

J. C. G. Andrae, T. Kovács

S1. Tables

Table S1.1 Rate constants for revised and added reactions compared to model in 1

\begin{tabular}{|c|c|c|c|c|c|}
\hline Toluene & $A^{\mathrm{a}}$ & $n^{\mathrm{a}}$ & $E^{\mathrm{a}}$ & & Ref \\
\hline $\begin{array}{l}\text { C6H5CH3 }(+\mathrm{M})<=> \\
\text { C6H5CH2+H(+M) }\end{array}$ & $2.78 \mathrm{E}+15$ & 0.17 & $9.1168 \mathrm{E}+04$ & & 2 \\
\hline LOW & $1.00 \mathrm{E}+98$ & -22.855 & $9.9882 \mathrm{E}+04$ & & \\
\hline TROE & $6.547194 \mathrm{E}-02$ & $1.511253 \mathrm{E}+01$ & $9.999996 \mathrm{E}+09$ & $7.596123 \mathrm{E}+07$ & \\
\hline $\begin{array}{l}\text { C6H5CH3 }(+\mathrm{M})<=> \\
\mathrm{C} 6 \mathrm{H} 5+\mathrm{CH} 3(+\mathrm{M})\end{array}$ & $1.95 \mathrm{E}+27$ & -3.16 & $1.07447 \mathrm{E}+05$ & & 2 \\
\hline LOW & $1.00 \mathrm{E}+98$ & -22.966 & $1.2208 \mathrm{E}+05$ & & \\
\hline TROE & 7.054562E-01 & $9.999989 \mathrm{E}+09$ & $4.599180 \mathrm{E}+02$ & $8.213938 \mathrm{E}+09$ & \\
\hline $\begin{array}{l}\mathrm{C} 6 \mathrm{H} 5 \mathrm{CH} 2+\mathrm{HO} 2<=> \\
\mathrm{C} 6 \mathrm{H} 5 \mathrm{CH} 2 \mathrm{O}+\mathrm{OH}\end{array}$ & $1.190 \mathrm{e}+09$ & 1.030 & -2249.04 & & 3 \\
\hline $\begin{array}{l}\mathrm{C} 6 \mathrm{H} 5 \mathrm{CH} 2 \mathrm{O}<=> \\
\text { C6H5+CH2O } \\
\text { DUP }\end{array}$ & $7.205 E+33$ & -6.21 & 36807 & & 4 \\
\hline $\begin{array}{l}\mathrm{C} 6 \mathrm{H} 5 \mathrm{CH} 2 \mathrm{O}<=> \\
\text { C6H5+CH2O } \\
\text { DUP }\end{array}$ & $1.073 \mathrm{E}+14$ & 0.16 & 31071 & & 4 \\
\hline $\begin{array}{l}\mathrm{C} 6 \mathrm{H} 5 \mathrm{CH} 2 \mathrm{O}<=> \\
\text { C6H5CHO+H } \\
\text { DUP }\end{array}$ & $5.227 \mathrm{E}+28$ & -5.08 & 22249 & & 4 \\
\hline $\begin{array}{l}\mathrm{C} 6 \mathrm{H} 5 \mathrm{CH} 2 \mathrm{O}<=> \\
\mathrm{C} 6 \mathrm{H} 5 \mathrm{CHO}+\mathrm{H} \\
\text { DUP }\end{array}$ & $5.07 \mathrm{E}+08$ & 1.56 & 16850 & & 4 \\
\hline $\begin{array}{l}\text { C6H5CH2OO(+M) } \\
<=> \\
\text { C6H5CH2+O2(+M) } \\
\text { LOW } \\
\text { TROE }\end{array}$ & $\begin{array}{r}1.797 \mathrm{E}-06 \\
6.114198 \mathrm{E}-01\end{array}$ & $\begin{array}{r}5.40 \\
9.999806 \mathrm{E}+09\end{array}$ & $\begin{array}{r}32200 \\
-7.63 \mathrm{E}+03 \\
1.037924 \mathrm{E}+00\end{array}$ & $1.786062 \mathrm{E}+09$ & 5,6 \\
\hline $\begin{array}{l}\mathrm{C} 6 \mathrm{H} 5 \mathrm{CH} 3+\mathrm{C} 6 \mathrm{H} 5 \mathrm{O} \\
<=> \\
\mathrm{C} 6 \mathrm{H} 5 \mathrm{CH} 2+\mathrm{C} 6 \mathrm{H} 5 \mathrm{OH}\end{array}$ & $1.6 \mathrm{E}+11$ & 0.0 & 15100 & & 7 \\
\hline $\begin{array}{l}\mathrm{C} 6 \mathrm{H} 5 \mathrm{CH} 3+\mathrm{C} 6 \mathrm{H} 4 \mathrm{CH} 3 \\
<=> \\
\mathrm{C} 6 \mathrm{H} 5 \mathrm{CH} 2+\mathrm{C} 6 \mathrm{H} 5 \mathrm{CH} 3\end{array}$ & $7.9 \mathrm{E}+13$ & 0.0 & 12000 & & 7 \\
\hline
\end{tabular}




\begin{tabular}{|c|c|c|c|c|}
\hline $\begin{array}{l}\mathrm{C} 6 \mathrm{H} 5 \mathrm{CH} 3+ \\
\mathrm{C} 6 \mathrm{H} 5 \mathrm{CH} 2 \mathrm{OO}<=> \\
\mathrm{C} 6 \mathrm{H} 5 \mathrm{CH} 2 \\
+\mathrm{C} 6 \mathrm{H} 5 \mathrm{CH} 2 \mathrm{OOH}\end{array}$ & $4.0 \mathrm{E}+11$ & 0.0 & 14000 & 7 \\
\hline $\begin{array}{l}\mathrm{C} 6 \mathrm{H} 5 \mathrm{CH} 3+ \\
\mathrm{C} 6 \mathrm{H} 5 \mathrm{CH} 2 \mathrm{O}<=> \\
\mathrm{C} 6 \mathrm{H} 5 \mathrm{CH} 2 \\
+\mathrm{C} 6 \mathrm{H} 5 \mathrm{CH} 2 \mathrm{OH}\end{array}$ & $1.6 \mathrm{E}+11$ & 0.0 & 11100 & 7 \\
\hline $\begin{array}{l}\mathrm{C} 6 \mathrm{H} 5 \mathrm{CH} 2 \mathrm{OO}<=> \\
\mathrm{C} 6 \mathrm{H} 5 \mathrm{CHO}+\mathrm{OH}\end{array}$ & $1.4 \mathrm{E}+10$ & 1.0 & 37500 & 6 \\
\hline $\begin{array}{l}\mathrm{C} 6 \mathrm{H} 5 \mathrm{CH} 2 \mathrm{OO}+\mathrm{H}<=> \\
\mathrm{C} 6 \mathrm{H} 5 \mathrm{CH} 2 \mathrm{OOH}\end{array}$ & $1.0 \mathrm{E}+14$ & 0.0 & 0 & 7 \\
\hline $\begin{array}{l}\mathrm{C} 6 \mathrm{H} 5 \mathrm{CH} 2 \mathrm{OO}+\mathrm{HO} 2 \\
<=> \\
\mathrm{C} 6 \mathrm{H} 5 \mathrm{CH} 2 \mathrm{OOH}+\mathrm{O} 2\end{array}$ & $2.0 \mathrm{E}+11$ & 0.0 & 0 & 7 \\
\hline $\begin{array}{l}2 \mathrm{C} 6 \mathrm{H} 5 \mathrm{CH} 2 \mathrm{OO}<=> \\
\mathrm{C} 6 \mathrm{H} 5 \mathrm{CH} 2 \mathrm{OH}+ \\
\mathrm{C} 6 \mathrm{H} 5 \mathrm{CHO}+\mathrm{O} 2\end{array}$ & $1.4 \mathrm{E}+10$ & 0.0 & -720 & 7 \\
\hline $\begin{array}{l}2 \mathrm{C} 6 \mathrm{H} 5 \mathrm{CH} 2 \mathrm{OO}<=> \\
2 \mathrm{C} 6 \mathrm{H} 5 \mathrm{CH} 2 \mathrm{O}+\mathrm{O} 2\end{array}$ & $6.3 \mathrm{E}+10$ & 0.0 & -720 & 7 \\
\hline $\begin{array}{l}\mathrm{C} 6 \mathrm{H} 5 \mathrm{CH} 2 \mathrm{O}+\mathrm{O} 2<=> \\
\mathrm{C} 6 \mathrm{H} 5 \mathrm{CHO}+\mathrm{HO} 2\end{array}$ & $6.0 \mathrm{E}+10$ & 0.0 & 1600 & 7 \\
\hline $\begin{array}{l}\mathrm{C} 6 \mathrm{H} 5 \mathrm{CH} 2 \mathrm{OOH}<=> \\
\mathrm{C} 6 \mathrm{H} 5 \mathrm{CH} 2+\mathrm{HO} 2\end{array}$ & $5.901 \mathrm{E} 13$ & 0.30 & 56166 & 3 \\
\hline $\begin{array}{l}\mathrm{C} 6 \mathrm{H} 5 \mathrm{CH} 2 \mathrm{OOH}<=> \\
\mathrm{C} 6 \mathrm{H} 5 \mathrm{CHO}+\mathrm{H} 2 \mathrm{O}\end{array}$ & 7.446E8 & 1.19 & 46128 & 3 \\
\hline $\begin{array}{l}\mathrm{C} 6 \mathrm{H} 5 \mathrm{CH} 2 \mathrm{O}<=> \\
\mathrm{C} 6 \mathrm{H} 6+\mathrm{HCO}\end{array}$ & $2.304 \mathrm{E} 32$ & -6.09 & 28920 & 4 \\
\hline N-heptane & $A^{a}$ & $n^{a}$ & $E^{a}$ & Ref \\
\hline $\begin{array}{l}\mathrm{C} 7 \mathrm{H} 15 \mathrm{O} 2=> \\
\mathrm{C} 7 \mathrm{H} 15-2+\mathrm{O} 2\end{array}$ & $2.300 \mathrm{E} 15$ & 0.00 & 26960 & This work \\
\hline $\begin{array}{l}\mathrm{OC} 7 \mathrm{H} 13 \mathrm{O} 2 \mathrm{H}=> \\
\mathrm{OC} 7 \mathrm{H} 13 \mathrm{O}+\mathrm{OH}\end{array}$ & $2.000 \mathrm{E} 15$ & 0.00 & 43020 & This work \\
\hline $\begin{array}{l}\mathrm{C} 7 \mathrm{H} 15-2=> \\
\text { PC4H9+CH2CHCH3 }\end{array}$ & $1.500 \mathrm{E} 13$ & 0.00 & 28203 & This work \\
\hline
\end{tabular}




\begin{tabular}{|c|c|c|c|c|}
\hline Iso-octane & $A^{a}$ & $n^{a}$ & $E^{a}$ & Ref \\
\hline $\begin{array}{l}\mathrm{C} 8 \mathrm{H} 18+\mathrm{O} 2<=> \\
\mathrm{AC} 8 \mathrm{H} 17+\mathrm{HO} 2\end{array}$ & $3.60 \mathrm{E}+14$ & 0.0 & 52800 & 8 \\
\hline $\begin{array}{l}\text { AC } 8 \mathrm{H} 17 \mathrm{OO}<=> \\
\text { AC } 8 \mathrm{H} 16 \mathrm{OOH}-\mathrm{B}\end{array}$ & $1.14 \mathrm{E}+11$ & 0.0 & 22175 & This work \\
\hline $\begin{array}{l}\mathrm{AC} 8 \mathrm{H} 16 \mathrm{OOH}-\mathrm{B}+\mathrm{O} 2 \\
<=> \\
\mathrm{AC} 8 \mathrm{H} 16 \mathrm{OOH}-\mathrm{BO} 2\end{array}$ & $1.86 \mathrm{E}+11$ & 0.0 & 0.0 & This work \\
\hline $\begin{array}{l}\text { AC } 8 \mathrm{H} 16 \mathrm{OOH}-\mathrm{BO} 2=> \\
\mathrm{OC} 8 \mathrm{H} 15 \mathrm{OOH}+\mathrm{OH}\end{array}$ & $1.20 \mathrm{E}+11$ & 0.0 & 17000 & This work \\
\hline $\begin{array}{l}\mathrm{C} 8 \mathrm{H} 18+\mathrm{OH}=> \\
\mathrm{AC} 8 \mathrm{H} 17+\mathrm{H} 2 \mathrm{O}\end{array}$ & $1.50 \mathrm{E}+13$ & 0.0 & 3000 & This work \\
\hline $\begin{array}{l}\mathrm{OC} 8 \mathrm{H} 15 \mathrm{OOH}=> \\
\mathrm{OC} 8 \mathrm{H} 15 \mathrm{O}+\mathrm{OH}\end{array}$ & $3.98 \mathrm{E}+16$ & 0.0 & 43000 & This work \\
\hline $\begin{array}{l}\mathrm{AC} 8 \mathrm{H} 17+\mathrm{O} 2<=> \\
\mathrm{JC} 8 \mathrm{H} 16+\mathrm{HO} 2\end{array}$ & $3.16 \mathrm{E}+11$ & 0.0 & 6400 & This work \\
\hline $\begin{array}{l}\mathrm{IC} 4 \mathrm{H} 8+\mathrm{O} 2<=> \\
\mathrm{IC} 4 \mathrm{H} 7+\mathrm{HO} 2\end{array}$ & $1.8693 \mathrm{E}+09$ & 1.3 & 40870 & 9 \\
\hline $\begin{array}{l}\mathrm{C} 8 \mathrm{H} 18+\mathrm{HO} 2<=> \\
\mathrm{AC} 8 \mathrm{H} 17+\mathrm{H} 2 \mathrm{O} 2\end{array}$ & $1.12 \mathrm{E}+13$ & 0.0 & 16950 & 10 \\
\hline $\begin{array}{l}\text { C8H18 }<> \\
\text { AC } 8 \mathrm{H} 17+\mathrm{H}\end{array}$ & $1.83 \mathrm{E}+55$ & -11.43 & 122500 & 11 \\
\hline $\begin{array}{l}\text { C8H18 }<=> \\
\text { NEOC5H11+I*C3H7 }\end{array}$ & $5.75 \mathrm{E}+46$ & -9.66 & 98040 & This work \\
\hline Cross reactions & $A^{a}$ & $n^{a}$ & $E^{a}$ & Ref \\
\hline $\begin{array}{l}\mathrm{C} 6 \mathrm{H} 5 \mathrm{CH} 2 \mathrm{OOH} \\
+\mathrm{C} 7 \mathrm{H} 15-1<=> \\
\text { C6H5CH2OO+C7H16 } \\
\text { DUP }\end{array}$ & $2.20 \mathrm{E}+11$ & 0.0 & 0 & This work ${ }^{\mathrm{b}}$ \\
\hline $\begin{array}{l}\mathrm{C} 6 \mathrm{H} 5 \mathrm{CH} 2 \mathrm{OOH}+ \\
\mathrm{C} 7 \mathrm{H} 15-2<=> \\
\text { C6H5CH2OO+C7H16 } \\
\text { DUP }\end{array}$ & $2.20 \mathrm{E}+11$ & 0.0 & 0 & This work ${ }^{\mathrm{b}}$ \\
\hline $\begin{array}{l}\text { C6H5CH2OOH + } \\
\text { AC8H17 <=> } \\
\text { C6H5CH2OO+C8H18 } \\
\text { DUP }\end{array}$ & $2.20 \mathrm{E}+11$ & 0.0 & 0 & This work $^{\mathrm{b}}$ \\
\hline
\end{tabular}

${ }^{\mathrm{a}} k_{\text {forward }}=A T^{n} \exp \left(-\frac{E}{R T}\right) ; A$ unit: mol-cm-s, $E$ unit: cal/mole; For reversible $(<=>$ ) reactions, reverse rate constants are calculated through the equilibrium constant and thermochemical data or otherwise explicitly stated.

${ }^{b}$ Duplicate reactions to $\mathrm{C}_{6} \mathrm{H}_{5} \mathrm{CH}_{2} \mathrm{OO}+\mathrm{RH}=\mathrm{R}+\mathrm{C} 6 \mathrm{H}_{5} \mathrm{CH}_{2} \mathrm{OOH}+\mathrm{R}$ 
Table S1.2 Rate constants for 2,4,4-trimethyl-1-pentene (JC8H16) reactions ${ }^{12}$

\begin{tabular}{|c|c|c|c|}
\hline & $A^{a}$ & $n^{a}$ & $E^{a}$ \\
\hline$=\mathrm{TC} 4 \mathrm{H} 9+\mathrm{IC} 4 \mathrm{H} 7$ & $1.13 E+92$ & -21.99 & 109815 . \\
\hline$=\mathrm{XC} 7 \mathrm{H} 13-\mathrm{Y} 2+\mathrm{CH} 3$ & $2.13 E+108$ & -26.53 & 135011. \\
\hline$=\mathrm{JC} 8 \mathrm{H} 15-\mathrm{B}+\mathrm{H}$ & $3.52 \mathrm{E}+92$ & -22.80 & 129512 . \\
\hline$=\mathrm{JC} 8 \mathrm{H} 15-\mathrm{D}+\mathrm{H}$ & $2.58 \mathrm{E}+94$ & -23.12 & 132883. \\
\hline $\mathrm{JC} 8 \mathrm{H} 16=\mathrm{IC} 4 \mathrm{H} 8+\mathrm{IC} 4 \mathrm{H} 8$ & $3.96 \mathrm{E}+27$ & -5.02 & 53633 \\
\hline $\mathrm{JC} 8 \mathrm{H} 16+\mathrm{O} 2=\mathrm{JC} 8 \mathrm{H} 15-\mathrm{B}+\mathrm{HO} 2$ & $6.20 \mathrm{E}+08$ & 1.301 & 38339.0 \\
\hline $\mathrm{JC} 8 \mathrm{H} 16+\mathrm{O} 2=\mathrm{JC} 8 \mathrm{H} 15-\mathrm{D}+\mathrm{HO} 2$ & $9.30 \mathrm{E}+08$ & 1.301 & 40939.0 \\
\hline $\mathrm{JC} 8 \mathrm{H} 16+\mathrm{OH}=\mathrm{JC} 8 \mathrm{H} 15-\mathrm{A}+\mathrm{H} 2 \mathrm{O}$ & $2.63 E+07$ & 1.80 & 278.2 \\
\hline $\mathrm{JC} 8 \mathrm{H} 16+\mathrm{OH}=\mathrm{JC} 8 \mathrm{H} 15-\mathrm{B}+\mathrm{H} 2 \mathrm{O}$ & $3.17 \mathrm{E}+06$ & 2.00 & -1434.0 \\
\hline $\mathrm{JC} 8 \mathrm{H} 16+\mathrm{OH}=\mathrm{JC} 8 \mathrm{H} 15-\mathrm{D}+\mathrm{H} 2 \mathrm{O}$ & $3.00 E+06$ & 2.00 & -239.0 \\
\hline $\mathrm{JC} 8 \mathrm{H} 16+\mathrm{H}=\mathrm{JC} 8 \mathrm{H} 15-\mathrm{A}+\mathrm{H} 2$ & $7.34 \mathrm{E}+05$ & 2.77 & 8147.0 \\
\hline $\mathrm{JC} 8 \mathrm{H} 16+\mathrm{H}=\mathrm{JC} 8 \mathrm{H} 15-\mathrm{B}+\mathrm{H} 2$ & $5.02 \mathrm{E}+04$ & 2.50 & -1912.0 \\
\hline $\mathrm{JC} 8 \mathrm{H} 16+\mathrm{H}=\mathrm{JC} 8 \mathrm{H} 15-\mathrm{D}+\mathrm{H} 2$ & $1.89 \mathrm{E}+05$ & 2.50 & 2390.1 \\
\hline $\mathrm{JC} 8 \mathrm{H} 16+\mathrm{HO} 2=\mathrm{JC} 8 \mathrm{H} 15-\mathrm{A}+\mathrm{H} 2 \mathrm{O} 2$ & $8.10 \mathrm{E}+04$ & 2.50 & 16690.8 \\
\hline $\mathrm{JC} 8 \mathrm{H} 16+\mathrm{HO} 2=\mathrm{JC} 8 \mathrm{H} 15-\mathrm{B}+\mathrm{H} 2 \mathrm{O} 2$ & $6.80 \mathrm{E}+03$ & 2.50 & 10113.8 \\
\hline $\mathrm{JC} 8 \mathrm{H} 16+\mathrm{HO} 2=\mathrm{JC} 8 \mathrm{H} 15-\mathrm{D}+\mathrm{H} 2 \mathrm{O} 2$ & $1.02 \mathrm{E}+04$ & 2.50 & 12339.3 \\
\hline $\mathrm{JC} 8 \mathrm{H} 16+\mathrm{CH} 3=\mathrm{JC} 8 \mathrm{H} 15-\mathrm{A}+\mathrm{CH} 4$ & $4.26 \mathrm{E}-14$ & 8.06 & 4154.0 \\
\hline $\mathrm{JC} 8 \mathrm{H} 16+\mathrm{CH} 3=\mathrm{JC} 8 \mathrm{H} 15-\mathrm{B}+\mathrm{CH} 4$ & $1.00 \mathrm{E}-01$ & 3.50 & 4046.1 \\
\hline $\mathrm{JC} 8 \mathrm{H} 16+\mathrm{CH} 3=\mathrm{JC} 8 \mathrm{H} 15-\mathrm{D}+\mathrm{CH} 4$ & $1.50 E-01$ & 3.50 & 5736.1 \\
\hline $\mathrm{JC} 8 \mathrm{H} 16+\mathrm{CH} 3 \mathrm{O}=\mathrm{JC} 8 \mathrm{H} 15-\mathrm{A}+\mathrm{CH} 3 \mathrm{OH}$ & $4.74 \mathrm{E}+11$ & 0.00 & 7000.0 \\
\hline $\mathrm{JC} 8 \mathrm{H} 16+\mathrm{CH} 3 \mathrm{O}=\mathrm{JC} 8 \mathrm{H} 15-\mathrm{B}+\mathrm{CH} 3 \mathrm{OH}$ & $4.23 E+10$ & 0.00 & 2400.0 \\
\hline $\mathrm{JC} 8 \mathrm{H} 16+\mathrm{CH} 3 \mathrm{O}=\mathrm{JC} 8 \mathrm{H} 15-\mathrm{D}+\mathrm{CH} 3 \mathrm{OH}$ & $6.35 E+10$ & 0.00 & 3000.0 \\
\hline $\mathrm{JC} 8 \mathrm{H} 16+\mathrm{C} 2 \mathrm{H} 3=\mathrm{JC} 8 \mathrm{H} 15-\mathrm{A}+\mathrm{C} 2 \mathrm{H} 4$ & $1.50 \mathrm{E}+12$ & 0.00 & 18000.0 \\
\hline $\mathrm{JC} 8 \mathrm{H} 16+\mathrm{C} 2 \mathrm{H} 3=\mathrm{JC} 8 \mathrm{H} 15-\mathrm{B}+\mathrm{C} 2 \mathrm{H} 4$ & $1.48 \mathrm{E}+00$ & 3.50 & 4281.4 \\
\hline $\mathrm{JC} 8 \mathrm{H} 16+\mathrm{C} 2 \mathrm{H} 3=\mathrm{JC} 8 \mathrm{H} 15-\mathrm{D}+\mathrm{C} 2 \mathrm{H} 4$ & $2.22 \mathrm{E}+00$ & 3.50 & 4681.4 \\
\hline $\mathrm{JC} 8 \mathrm{H} 16+\mathrm{C} 2 \mathrm{H} 5=\mathrm{JC} 8 \mathrm{H} 15-\mathrm{A}+\mathrm{C} 2 \mathrm{H} 6$ & $1.50 \mathrm{E}+11$ & 0.00 & 13400.0 \\
\hline $\mathrm{JC} 8 \mathrm{H} 16+\mathrm{C} 2 \mathrm{H} 5=\mathrm{JC} 8 \mathrm{H} 15-\mathrm{B}+\mathrm{C} 2 \mathrm{H} 6$ & $2.23 E+00$ & 3.50 & 6236.6 \\
\hline $\mathrm{JC} 8 \mathrm{H} 16+\mathrm{C} 2 \mathrm{H} 5=\mathrm{JC} 8 \mathrm{H} 15-\mathrm{D}+\mathrm{C} 2 \mathrm{H} 6$ & $6.68 \mathrm{E}+00$ & 3.50 & 6636.6 \\
\hline $\mathrm{IC} 4 \mathrm{H} 8+\mathrm{IC} 4 \mathrm{H} 7=\mathrm{JC} 8 \mathrm{H} 15-\mathrm{A}$ & $2.92 E+03$ & 2.36 & 11860.0 \\
\hline $\mathrm{DMPD} 13+\mathrm{CH} 3=\mathrm{JC} 8 \mathrm{H} 15-\mathrm{B}$ & $2.92 E+03$ & 2.36 & 8360.0 \\
\hline $\mathrm{NEOC5H11}+\mathrm{H} 3 \mathrm{CCCH}=\mathrm{JC} 8 \mathrm{H} 15-\mathrm{D}$ & $1.50 \mathrm{E}+11$ & 0.0 & 4970.0 \\
\hline $\mathrm{JC} 8 \mathrm{H} 15-\mathrm{A}=\mathrm{JC} 8 \mathrm{H} 15-\mathrm{D}$ & $1.10 E+13$ & -0.6 & 7500.0 \\
\hline $\mathrm{JC} 8 \mathrm{H} 15-\mathrm{B}+\mathrm{HO} 2=\mathrm{JC} 8 \mathrm{H} 15 \mathrm{O}-\mathrm{B}+\mathrm{OH}$ & $3.50 E+12$ & 0.0 & -1000.0 \\
\hline $\mathrm{JC} 8 \mathrm{H} 15-\mathrm{D}+\mathrm{HO} 2=\mathrm{JC} 8 \mathrm{H} 15 \mathrm{O}-\mathrm{D}+\mathrm{OH}$ & $7.00 \mathrm{E}+12$ & 0.0 & -1000.0 \\
\hline $\mathrm{IC} 3 \mathrm{H} 5 \mathrm{CHO}+\mathrm{TC} 4 \mathrm{H} 9=\mathrm{JC} 8 \mathrm{H} 150-\mathrm{B}$ & $7.49 E+22$ & -2.8 & 21140.0 \\
\hline $\mathrm{NEOC} 5 \mathrm{H} 11 \mathrm{CCH} 2+\mathrm{CH} 2 \mathrm{O}=\mathrm{JC} 8 \mathrm{H} 150-\mathrm{D}$ & $1.50 E+11$ & 0.0 & 10600.0 \\
\hline $\mathrm{NEOC} 5 \mathrm{H} 11 \mathrm{CCH} 2+\mathrm{O} 2=\mathrm{NEOC} 5 \mathrm{H} 11 \mathrm{CO}+\mathrm{CH} 2 \mathrm{O}$ & $4.34 \mathrm{E}+12$ & 0.0 & 0.0 \\
\hline $\mathrm{H} 3 \mathrm{CCCH}+\mathrm{TC} 4 \mathrm{H} 9=\mathrm{NEOC} 5 \mathrm{H} 11 \mathrm{CCH} 2$ & $8.80 \mathrm{E}+03$ & 2.48 & 6130.0 \\
\hline $\mathrm{CH} 2 \mathrm{CHC} . \mathrm{H} 2+\mathrm{IC} 4 \mathrm{H} 8=\mathrm{XC} 7 \mathrm{H} 13-\mathrm{Y} 2$ & $2.00 E+11$ & 0.0 & 2010.0 \\
\hline ! 2, 4-DI-METHYL-1,3-PENTADIENE (DMPD) & & & \\
\hline $\mathrm{DMPD} 13+\mathrm{OH}=\mathrm{DMPD} 13-\mathrm{B}+\mathrm{H} 2 \mathrm{O}$ & $3.00 E+06$ & 2.0 & -239.0 \\
\hline $\mathrm{DMPD} 13+\mathrm{OH}=\mathrm{DMPD} 13-\mathrm{E}+\mathrm{H} 2 \mathrm{O}$ & $6.00 E+06$ & 2.0 & -239.0 \\
\hline $\mathrm{DMPD} 13+\mathrm{H}=\operatorname{DMPD} 13-\mathrm{B}+\mathrm{H} 2$ & $1.89 \mathrm{E}+05$ & 2.5 & 2390.1 \\
\hline $\mathrm{DMPD} 13+\mathrm{H}=\operatorname{DMPD} 13-\mathrm{E}+\mathrm{H} 2$ & $3.79 E+05$ & 2.5 & 2390.1 \\
\hline $\mathrm{DMPD} 13+\mathrm{HO} 2=\mathrm{DMPD} 13-\mathrm{B}+\mathrm{H} 2 \mathrm{O} 2$ & $1.02 \mathrm{E}+04$ & 2.5 & 12339.3 \\
\hline $\mathrm{DMPD} 13+\mathrm{HO} 2=\mathrm{DMPD} 13-\mathrm{E}+\mathrm{H} 2 \mathrm{O} 2$ & $2.04 E+04$ & 2.5 & 12339.3 \\
\hline $\mathrm{DMPD} 13+\mathrm{CH} 3=\mathrm{DMPD} 13-\mathrm{B}+\mathrm{CH} 4$ & $1.50 E-01$ & 3.5 & 5736.1 \\
\hline DMPD13 $+\mathrm{CH} 3=\operatorname{DMPD} 13-\mathrm{E}+\mathrm{CH} 4$ & $3.01 E-01$ & 3.5 & 5736.1 \\
\hline $\mathrm{DMPD} 13+\mathrm{H}=\mathrm{CH} 2 \mathrm{CHCH} 3+\mathrm{IC} 4 \mathrm{H} 7-\mathrm{I} 1$ & $7.30 E+29$ & -4.34 & 21647.0 \\
\hline $\mathrm{DMPD} 13+\mathrm{H}=\mathrm{IC} 4 \mathrm{H} 8+\mathrm{CH} 2 \mathrm{CHC} . \mathrm{H} 2$ & $7.30 E+29$ & -4.34 & 21647.0 \\
\hline \multicolumn{4}{|l|}{ ! CONSUMPTION OF DMPD RADICALS: } \\
\hline $\mathrm{H} 3 \mathrm{CCCH}+\mathrm{IC} 4 \mathrm{H} 7-\mathrm{I} 1=\mathrm{DMPD} 13-\mathrm{B}$ & $2.00 E+11$ & 0.0 & 2010.0 \\
\hline $\mathrm{MPT} 124+\mathrm{CH} 3=\operatorname{DMPD} 13-\mathrm{E}$ & $1.50 \mathrm{E}+11$ & 0.0 & 4970.0 \\
\hline ! CONSUMPTION OF MPT124 (4-METHYL-1, & $2,4-\mathrm{PENTATH}$ & $\mathrm{JE} O \mathrm{OR} \mathrm{C}^{\star} \mathrm{C}$ & $C(C) C * C * C)$ \\
\hline $\operatorname{MPT} 124+\mathrm{OH}=\operatorname{MPT} 124 \mathrm{~J}+\mathrm{H} 2 \mathrm{O}$ & $3.00 \mathrm{E}+06$ & 2.0 & -239.0 \\
\hline $\operatorname{MPT} 124+\mathrm{H}=\mathrm{MPT} 124 \mathrm{~J}+\mathrm{H} 2$ & $1.89 \mathrm{E}+05$ & 2.5 & 2390.1 \\
\hline $\operatorname{MPT} 124+\mathrm{HO} 2=\operatorname{MPT} 124 \mathrm{~J}+\mathrm{H} 2 \mathrm{O} 2$ & $1.02 \mathrm{E}+04$ & 2.5 & 12339.3 \\
\hline $\mathrm{MPT} 124+\mathrm{CH} 3=\mathrm{MPT} 124 \mathrm{~J}+\mathrm{CH} 4$ & $1.50 \mathrm{E}-01$ & 3.5 & 5736.1 \\
\hline
\end{tabular}




\begin{tabular}{|c|c|c|c|}
\hline \multirow[b]{2}{*}{$!$ CONSUMPTION OF MPT124J OR $\mathrm{C} * \mathrm{C}(\mathrm{C}.) \mathrm{C} * \mathrm{C} * \mathrm{C}$} & $A^{a}$ & $n^{a}$ & $E^{a}$ \\
\hline & & & \\
\hline $\begin{array}{l}\mathrm{H} 2 \mathrm{CCCH}+\mathrm{H} 3 \mathrm{CCCH}=\mathrm{MPT} 124 \mathrm{~J} \\
! \operatorname{Ref}[11]\end{array}$ & $8.50 \mathrm{E}+10$ & 0.0 & 1300.0 \\
\hline NEOC5H11CO $=$ NEOC5H11+CO & $2.433 \mathrm{E}+17$ & -1.27 & $1.344 \mathrm{E}+04$ \\
\hline $\mathrm{NEOC} 5 \mathrm{H} 11 \mathrm{CO}=\mathrm{TC} 4 \mathrm{H} 9+\mathrm{CH} 2 \mathrm{CO}$ & $3.657 \mathrm{E}+16$ & -1.03 & $3.487 \mathrm{E}+04$ \\
\hline ! Ref [11] & & & \\
\hline $\mathrm{IC} 4 \mathrm{H} 7-\mathrm{II}=\mathrm{H} 3 \mathrm{CCCH}+\mathrm{CH} 3$ & \multicolumn{3}{|c|}{ 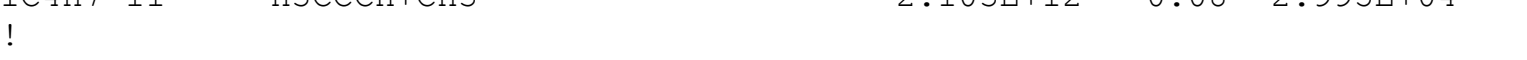 } \\
\hline \multicolumn{4}{|l|}{ ! IC4H8 reactions } \\
\hline $\mathrm{TC} 4 \mathrm{H} 9=\mathrm{H}+\mathrm{IC} 4 \mathrm{H} 8$ & $4.650 \mathrm{E}+46$ & -9.83 & $5.508 \mathrm{E}+04$ \\
\hline $\mathrm{IC} 4 \mathrm{H} 8+\mathrm{H}=\mathrm{IC} 4 \mathrm{H} 7+\mathrm{H} 2$ & $3.400 \mathrm{E}+05$ & 2.50 & $2.492 \mathrm{E}+03$ \\
\hline $\mathrm{IC} 4 \mathrm{H} 8+\mathrm{OH}=\mathrm{IC} 4 \mathrm{H} 7+\mathrm{H} 2 \mathrm{O}$ & $5.200 \mathrm{E}+06$ & 2.00 & $-2.980 \mathrm{E}+02$ \\
\hline $\mathrm{IC} 4 \mathrm{H} 8=\mathrm{IC} 4 \mathrm{H} 7+\mathrm{H}$ & $3.070 \mathrm{E}+55$ & -11.49 & $1.143 \mathrm{E}+05$ \\
\hline $\mathrm{IC} 4 \mathrm{H} 7+\mathrm{HO} 2=\mathrm{IC} 4 \mathrm{H} 7 \mathrm{O}+\mathrm{OH}$ & $7.000 \mathrm{E}+12$ & 0.00 & $-1.000 \mathrm{E}+03$ \\
\hline $\mathrm{IC} 4 \mathrm{H} 7 \mathrm{O}=\mathrm{IC} 3 \mathrm{H} 5 \mathrm{CHO}+\mathrm{H}$ & $5.000 \mathrm{E}+13$ & 0.00 & $2.910 \mathrm{E}+04$ \\
\hline $\begin{array}{l}\text { IC } 4 \mathrm{H} 7 \mathrm{O}=\mathrm{IC} 3 \mathrm{H} 5 \mathrm{CHO}+\mathrm{H} \\
\mathrm{IC} 4 \mathrm{H} 7+\mathrm{O}=\mathrm{IC} 3 \mathrm{H} 5 \mathrm{CHO}+\mathrm{H}\end{array}$ & $6.030 \mathrm{E}+13$ & 0.00 & $0.000 \mathrm{E}+00$ \\
\hline $\mathrm{IC} 4 \mathrm{H} 8+\mathrm{O}=\mathrm{CH} 2 \mathrm{CO}+\mathrm{CH} 3+\mathrm{CH} 3$ & $3.330 \mathrm{E}+07$ & 1.76 & $7.600 \mathrm{E}+01$ \\
\hline $\mathrm{IC} 4 \mathrm{H} 8+\mathrm{H}=\mathrm{CH} 2 \mathrm{CHCH} 3+\mathrm{CH} 3$ & $5.680 \mathrm{E}+33$ & -5.72 & $2.000 \mathrm{E}+04$ \\
\hline \multirow{2}{*}{$! \mathrm{IC} 4 \mathrm{H} 7=\mathrm{H} 2 \mathrm{CCCH} 2+\mathrm{CH} 3$} & $1.230 \mathrm{E}+47$ & -9.74 & $7.426 \mathrm{E}+04$ \\
\hline & \multicolumn{3}{|c|}{ ! k_inf.: Zheng et al., J Phys Chem A, 2005 [13]. } \\
\hline $\begin{array}{l}\text { IC4H7 }(+\mathrm{M})=\mathrm{H} 2 \mathrm{CCCH} 2+\mathrm{CH} 3(+\mathrm{M}) \\
\text { LOW } / 2.0 \mathrm{e} 141.3856360 / \text { ! This work }\end{array}$ & $7.1 \mathrm{e} 10$ & 1.38 & 56360 \\
\hline $\mathrm{IC} 4 \mathrm{H} 7 \mathrm{O}=\mathrm{CH} 2 \mathrm{CHC} \cdot \mathrm{H} 2+\mathrm{CH} 2 \mathrm{O}$ & $2.925 \mathrm{E}+21$ & -2.39 & $3.537 \mathrm{E}+04$ \\
\hline $\mathrm{IC} 3 \mathrm{H} 5 \mathrm{CHO}+\mathrm{OH}=\mathrm{IC} 3 \mathrm{H} 5 \mathrm{CO}+\mathrm{H} 2 \mathrm{O}$ & $2.690 \mathrm{E}+10$ & 0.76 & $-3.400 \mathrm{E}+02$ \\
\hline $\mathrm{IC} 3 \mathrm{H} 5 \mathrm{CHO}+\mathrm{CH} 3=\mathrm{IC} 3 \mathrm{H} 5 \mathrm{CO}+\mathrm{CH} 4$ & $3.980 \mathrm{E}+12$ & 0.00 & $8.700 \mathrm{E}+03$ \\
\hline $\mathrm{IC} 3 \mathrm{H} 5 \mathrm{CHO}+\mathrm{O}=\mathrm{IC} 3 \mathrm{H} 5 \mathrm{CO}+\mathrm{OH}$ & $7.180 \mathrm{E}+12$ & 0.00 & $1.389 \mathrm{E}+03$ \\
\hline $\mathrm{IC} 3 \mathrm{H} 5 \mathrm{CHO}+\mathrm{H}=I \mathrm{C} 3 \mathrm{H} 5 \mathrm{CO}+\mathrm{H} 2$ & $2.600 \mathrm{E}+12$ & 0.00 & $2.600 \mathrm{E}+03$ \\
\hline $\mathrm{IC} 3 \mathrm{H} 5 \mathrm{CO}=\mathrm{CH} 2 \mathrm{CHC} \cdot \mathrm{H} 2+\mathrm{CO}$ & $1.278 \mathrm{E}+20$ & -1.89 & $3.446 \mathrm{E}+04$ \\
\hline $\mathrm{IC} 4 \mathrm{H} 7+\mathrm{O} 2=\mathrm{IC} 3 \mathrm{H} 5 \mathrm{CHO}+\mathrm{OH}$ & $2.470 \mathrm{E}+13$ & -0.45 & $2.302 \mathrm{E}+04$ \\
\hline $\mathrm{IC} 4 \mathrm{H} 7+\mathrm{O} 2=\mathrm{H} 2 \mathrm{CCCH} 2+\mathrm{CH} 2 \mathrm{O}+\mathrm{OH}$ & $7.290 \mathrm{E}+29$ & -5.71 & $2.145 \mathrm{E}+04$ \\
\hline & & & \\
\hline ! Cross reactions from Ref [14] & & & \\
\hline$\dot{\mathrm{C}} 6 \mathrm{H} 5 \mathrm{CHO}+\mathrm{C} 7 \mathrm{H} 15-1=\mathrm{C} 6 \mathrm{H} 5 \mathrm{CO}+\mathrm{C} 7 \mathrm{H} 16$ & $1.30 \mathrm{e} 12$ & 0.0 & 7500 \\
\hline $\mathrm{C} 6 \mathrm{H} 5 \mathrm{CHO}+\mathrm{C} 7 \mathrm{H} 15-2=\mathrm{C} 6 \mathrm{H} 5 \mathrm{CO}+\mathrm{C} 7 \mathrm{H} 16$ & $1.30 e 12$ & 0.0 & 7500 \\
\hline $\mathrm{C} 6 \mathrm{H} 5 \mathrm{CHO}+\mathrm{AC} 8 \mathrm{H} 17=\mathrm{C} 6 \mathrm{H} 5 \mathrm{CO}+\mathrm{C} 8 \mathrm{H} 18$ & $1.30 \mathrm{e} 12$ & 0.0 & 7500 \\
\hline $\mathrm{C} 6 \mathrm{H} 5 \mathrm{CH} 3+\mathrm{IC} 4 \mathrm{H} 7=\mathrm{C} 6 \mathrm{H} 5 \mathrm{CH} 2+\mathrm{IC} 4 \mathrm{H} 8$ & $1.60 \mathrm{E}+12$ & 0.0 & 15100.0 \\
\hline $\mathrm{C} 6 \mathrm{H} 5 \mathrm{CH} 3+\mathrm{CH} 2 \mathrm{CHC} \cdot \mathrm{H} 2=\mathrm{C} 6 \mathrm{H} 5 \mathrm{CH} 2+\mathrm{CH} 2 \mathrm{CHCH} 3$ & $1.60 \mathrm{E}+12$ & 0.0 & 15100.0 \\
\hline $\mathrm{C} 6 \mathrm{H} 5 \mathrm{CH} 3+\mathrm{CH} 2 \mathrm{C} \cdot \mathrm{CH} 3=\mathrm{C} 6 \mathrm{H} 5 \mathrm{CH} 2+\mathrm{CH} 2 \mathrm{CHCH} 3$ & $1.60 \mathrm{E}+12$ & 0.0 & 11100.0 \\
\hline $\mathrm{C} 6 \mathrm{H} 5 \mathrm{CHO}+\mathrm{IC} 4 \mathrm{H} 7=\mathrm{C} 6 \mathrm{H} 5 \mathrm{CO}+\mathrm{IC} 4 \mathrm{H} 8$ & $1.30 \mathrm{E}+12$ & 0.0 & 11500.0 \\
\hline $\mathrm{C} 6 \mathrm{H} 5 \mathrm{CHO}+\mathrm{CH} 2 \mathrm{CHC} . \mathrm{H} 2=\mathrm{C} 6 \mathrm{H} 5 \mathrm{CO}+\mathrm{CH} 2 \mathrm{CHCH} 3$ & $1.30 \mathrm{E}+12$ & 0.0 & 11500.0 \\
\hline $\mathrm{C} 6 \mathrm{H} 5 \mathrm{CHO}+\mathrm{CH} 2 \mathrm{C} . \mathrm{CH} 3=\mathrm{C} 6 \mathrm{H} 5 \mathrm{CO}+\mathrm{CH} 2 \mathrm{CHCH} 3$ & $1.30 \mathrm{E}+12$ & 0.0 & 7500.0 \\
\hline $\mathrm{C} 6 \mathrm{H} 5 \mathrm{CHO}+\mathrm{C} 2 \mathrm{H} 3=\mathrm{C} 6 \mathrm{H} 5 \mathrm{CO}+\mathrm{C} 2 \mathrm{H} 4$ & $1.30 \mathrm{E}+12$ & 0.0 & 7500.0 \\
\hline $\mathrm{C} 6 \mathrm{H} 6+\mathrm{IC} 4 \mathrm{H} 7=\mathrm{C} 6 \mathrm{H} 5+\mathrm{IC} 4 \mathrm{H} 8$ & $6.30 \mathrm{E}+11$ & 0.0 & 20000.0 \\
\hline $\mathrm{C} 6 \mathrm{H} 6+\mathrm{CH} 2 \mathrm{CHC} \cdot \mathrm{H} 2=\mathrm{C} 6 \mathrm{H} 5+\mathrm{CH} 2 \mathrm{CHCH} 3$ & $6.30 \mathrm{E}+11$ & 0.0 & 20000.0 \\
\hline $\mathrm{C} 6 \mathrm{H} 6+\mathrm{CH} 2 \mathrm{C} . \mathrm{CH} 3=\mathrm{C} 6 \mathrm{H} 5+\mathrm{CH} 2 \mathrm{CHCH} 3$ & $6.30 \mathrm{E}+11$ & 0.0 & 15000.0 \\
\hline $\mathrm{C} 6 \mathrm{H} 6+\mathrm{C} 2 \mathrm{H} 3=\mathrm{C} 6 \mathrm{H} 5+\mathrm{C} 2 \mathrm{H} 4$ & $6.30 \mathrm{E}+11$ & 0.0 & 15000.0 \\
\hline & & & \\
\hline ! Cross reactions this work & & & \\
\hline & & & \\
\hline $\mathrm{JC} 8 \mathrm{H} 15-\mathrm{B}+\mathrm{C} 6 \mathrm{H} 5 \mathrm{CH} 3=\mathrm{C} 6 \mathrm{H} 5 \mathrm{CH} 2+\mathrm{JC} 8 \mathrm{H} 16$ & 1. $0 \mathrm{E}+11$ & 0.0 & 12000 \\
\hline $\mathrm{JC} 8 \mathrm{H} 15-\mathrm{B}+\mathrm{C} 7 \mathrm{H} 16=\mathrm{C} 7 \mathrm{H} 15-1+\mathrm{JC} 8 \mathrm{H} 16$ & $9.0 \mathrm{E}+11$ & 0.0 & 15000 \\
\hline $\mathrm{JC} 8 \mathrm{H} 15-\mathrm{B}+\mathrm{C} 7 \mathrm{H} 16=\mathrm{C} 7 \mathrm{H} 15-2+\mathrm{JC} 8 \mathrm{H} 16$ & $2.0 \mathrm{E}+11$ & 0.0 & 12500 \\
\hline $\mathrm{JC} 8 \mathrm{H} 15-\mathrm{D}+\mathrm{C} 7 \mathrm{H} 16=\mathrm{C} 7 \mathrm{H} 15-1+\mathrm{JC} 8 \mathrm{H} 16$ & $9.0 \mathrm{E}+11$ & 0.0 & 15000 \\
\hline $\mathrm{JC} 8 \mathrm{H} 15-\mathrm{D}+\mathrm{C} 7 \mathrm{H} 16=\mathrm{C} 7 \mathrm{H} 15-2+\mathrm{JC} 8 \mathrm{H} 16$ & $2.0 \mathrm{E}+11$ & 0.0 & 12500 \\
\hline $\mathrm{C} 8 \mathrm{H} 18+\mathrm{JC} 8 \mathrm{H} 15-\mathrm{B}=\mathrm{JC} 8 \mathrm{H} 16+\mathrm{AC} 8 \mathrm{H} 17$ & $9.0 \mathrm{E}+11$ & 0.0 & 13500 \\
\hline $\mathrm{C} 8 \mathrm{H} 18+\mathrm{JC} 8 \mathrm{H} 15-\mathrm{D}=\mathrm{JC} 8 \mathrm{H} 16+\mathrm{AC} 8 \mathrm{H} 17$ & $9.0 \mathrm{E}+11$ & 0.0 & 13500 \\
\hline
\end{tabular}




\begin{tabular}{|c|c|c|c|}
\hline Lemp & $A^{a}$ & $n^{a}$ & $E^{a}$ \\
\hline $\begin{array}{l}\mathrm{JC} 8 \mathrm{H} 15-\mathrm{B}+\mathrm{O} 2=\mathrm{JC} 8 \mathrm{H} 1500 \\
\mathrm{REV} / 2.51 \mathrm{e} 130.027400 /\end{array}$ & $2.00 \mathrm{E} 09$ & 0 & 0 \\
\hline $\begin{array}{l}\text { JC8H15-D+O2 = JC8H1500 } \\
\text { REV / } 2.51 e 130.027400\end{array}$ & $2.00 E 09$ & 0 & 0 \\
\hline $\begin{array}{l}\mathrm{JC} 8 \mathrm{H} 1500=\mathrm{JC} 8 \mathrm{H} 1400 \mathrm{H} \\
\mathrm{REV} / 2.00 \mathrm{e} 110.011000 /\end{array}$ & $2.00 \mathrm{E} 11$ & 0 & 26450 \\
\hline $\begin{array}{l}\mathrm{JC} 8 \mathrm{H} 140 \mathrm{OH}+\mathrm{O} 2=\mathrm{JC} 8 \mathrm{H} 140 \mathrm{OH}-\mathrm{O} 2 \\
\mathrm{REV} / 2.51 \mathrm{e} 130.027400 /\end{array}$ & $1.86 \mathrm{E} 11$ & 0 & 0 \\
\hline $\mathrm{JC} 8 \mathrm{H1} 40 \mathrm{OH}-\mathrm{O} 2 \Rightarrow \mathrm{OC} 8 \mathrm{H1} 30 \mathrm{OH}+\mathrm{OH}$ & $5.00 \mathrm{E} 10$ & 0 & 20450 \\
\hline $\mathrm{OC} 8 \mathrm{H} 130 \mathrm{OH}=>\mathrm{OC} 8 \mathrm{H} 13 \mathrm{O}+\mathrm{OH}$ & $3.00 \mathrm{E} 11$ & 0 & 22000 \\
\hline $\mathrm{OC} 8 \mathrm{H} 13 \mathrm{O}+\mathrm{O} 2=>\mathrm{C} 2 \mathrm{H}+2 \mathrm{CH} 2 \mathrm{O}+\mathrm{H} 2 \mathrm{CCCH} 2+\mathrm{CH} 3+\mathrm{HO} 2$ & $2.45 \mathrm{E} 13$ & 0 & 32000 \\
\hline
\end{tabular}

${ }^{\mathrm{a}} k_{\text {forward }}=A T^{n} \exp \left(-\frac{E}{R T}\right) ; A$ unit: mol-cm-s, $E$ unit: cal/mole; Rate constants for reversible $(=)$ reactions are calculated through the equilibrium constant and thermochemical data.

\section{$\underline{\text { S1.3 References }}$}

(1) Andrae, J. C. G. Fuel 2013, 107, 740-748.

(2) Klippenstein S. J.; Harding L. B.; Georgievskii, Y. Proc. Combust. Inst. 2007, 31, 221-229.

(3) da Silva, G.; Bozzelli J. W. Proc. Combust. Inst. 2009, 32, 287-294.

(4) da Silva, G.; Bozzelli J. W. J. Phys. Chem. A 2009, 113, 6979-6986.

(5) Murakami, Y.; Oguchi, T.; Hashimoto K.; Nosaka Y. J. Phys. Chem. A 2007, 111, $13200-13208$.

(6) Yuan, W.; Li, Y.; Dagaut, P.; Yang, J.; Qi, F. Combust. Flame 2015, 162, 3-21.

(7) Bounaceur, R.; da Costa, I.; Fournet, R.; Billaud, F.; Battin-Leclerc, F. Int. J. Chem. Kin. 2005, 37, 25-49.

(8) Cai, L.; Pitsch, H. Combust. Flame 2015, 162, 1623-1637.

(9) Chen C. J.; Bozzelli J. W. J. Phys. Chem. 2000, 104, 9715-9732.

(10) Wang, Y.; Yao, M.; Zheng, Z. Fuel 2013, 113, 347-356.

(11) Curran, H. J.; Gaffuri, P.; Pitz W. J.; Westbrook C. K. Combust. Flame 2002, 129, 253-280.

(12) Metcalfe W. K.; Pitz, W. J.; Curran, H. J.; Simmie, J. M.; Westbrook, C. K. Proc. Combust Inst 2007, 31, 377-384.

(13) Zheng, X. L.; Sun, H. Y.; Law, C. K. J. Phys. Chem. A 2005, 109, 9044-9053.

(14) Sakai, Y.; Miyoshi, A.; Koshi, M.; Pitz, W. J. Proc. Combust. Inst. 2009, 32, 411418. 
S2. Model validation

\section{$\underline{\text { S2.1 Validation for single component fuels }}$}

2,4,4-trimethyl-1-pentene (DIB-1)

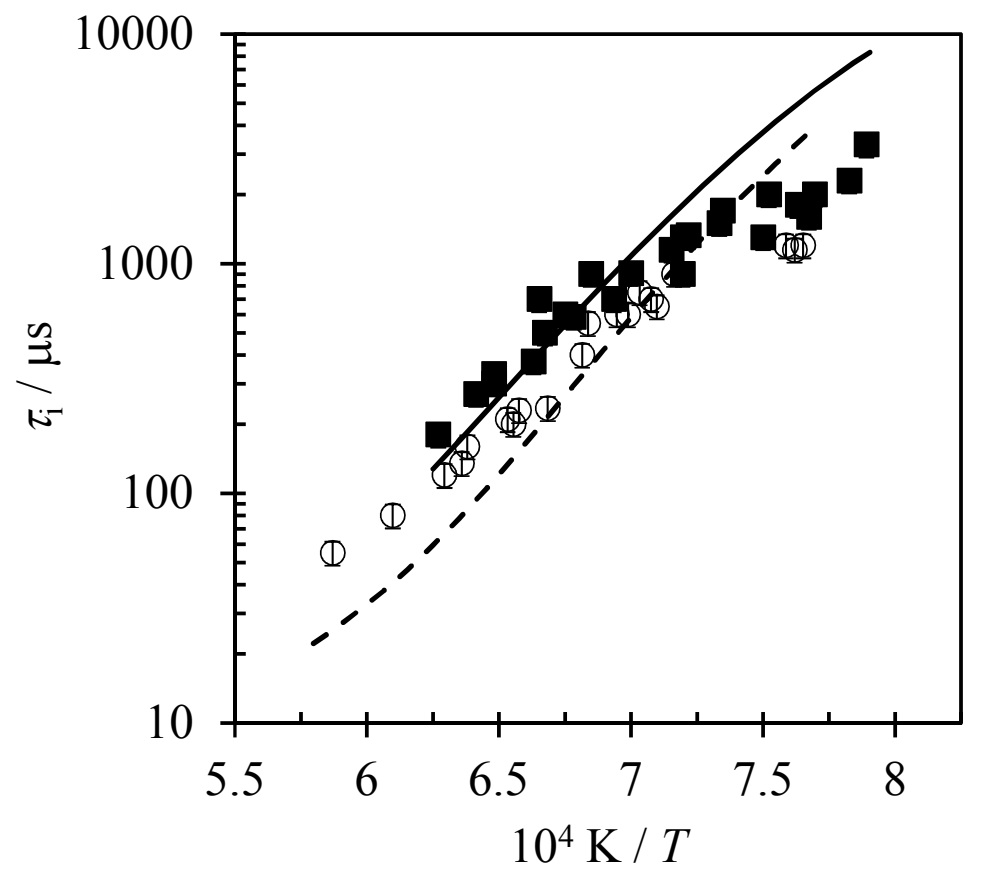

Fig. 1. Experimental (points) by Metcalfe et al. ${ }^{1}$ and model-predicted (lines) ignition delay times for 2,4,4-trimethyl-1-pentene oxidation behind reflected shock waves at $1.0 \mathrm{~atm}$ in $\mathrm{Ar}$, (匹) 0.75\% fuel, $\phi=1.0$, (○) $0.375 \%$ fuel, $\phi=0.5$. Dashed line corresponds to open symbols. Simulations with detailed model (159 species). 


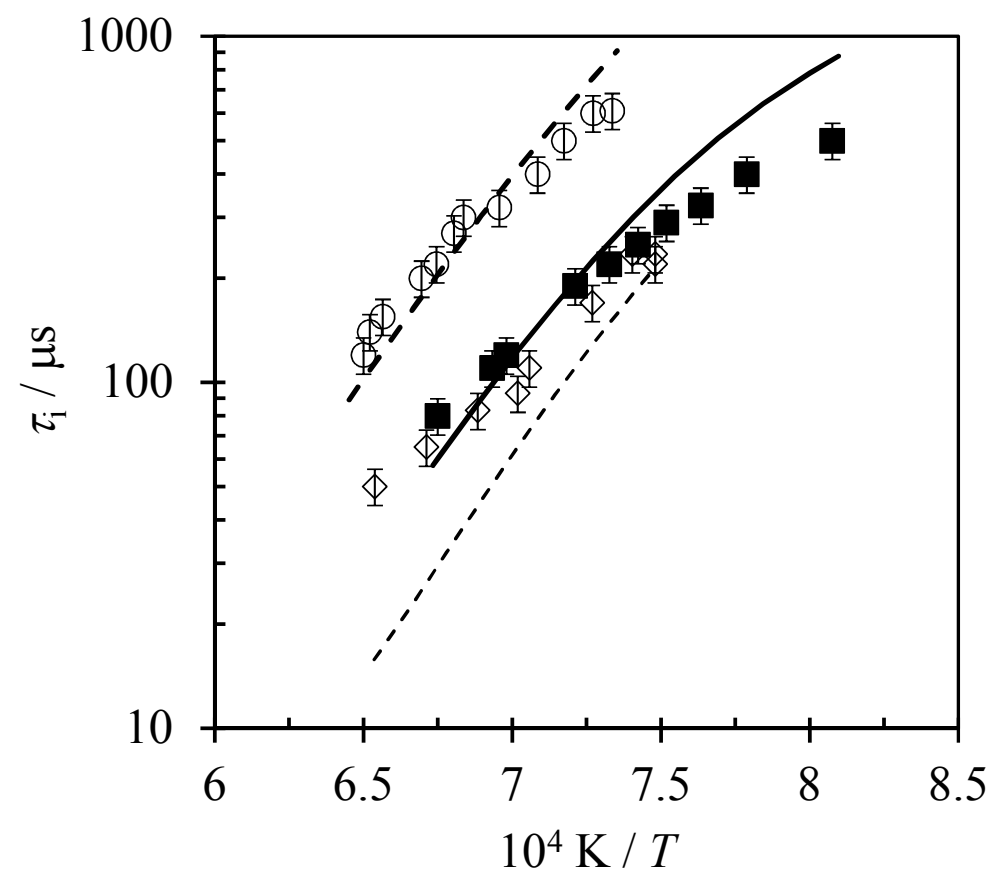

Fig. 2. Experimental (points) by Metcalfe et al. ${ }^{1}$ and model-predicted (lines) ignition delay times for 2,4,4-trimethyl-1-pentene oxidation behind reflected shock waves at $4.0 \mathrm{~atm}$ in $\mathrm{Ar},(\bigcirc) 0.75 \%$ fuel, $9.0 \%$ oxygen, $\phi=1.0$, ( $) 0.75 \%$ fuel, $18.0 \%$ oxygen, $\phi=0.5$, ( $\diamond) 0.375 \%$ fuel, $18.0 \%$ oxygen, $\phi=0.25$. Dashed lines correspond to open symbols. Simulations with detailed model (159 species). 


\section{Toluene}

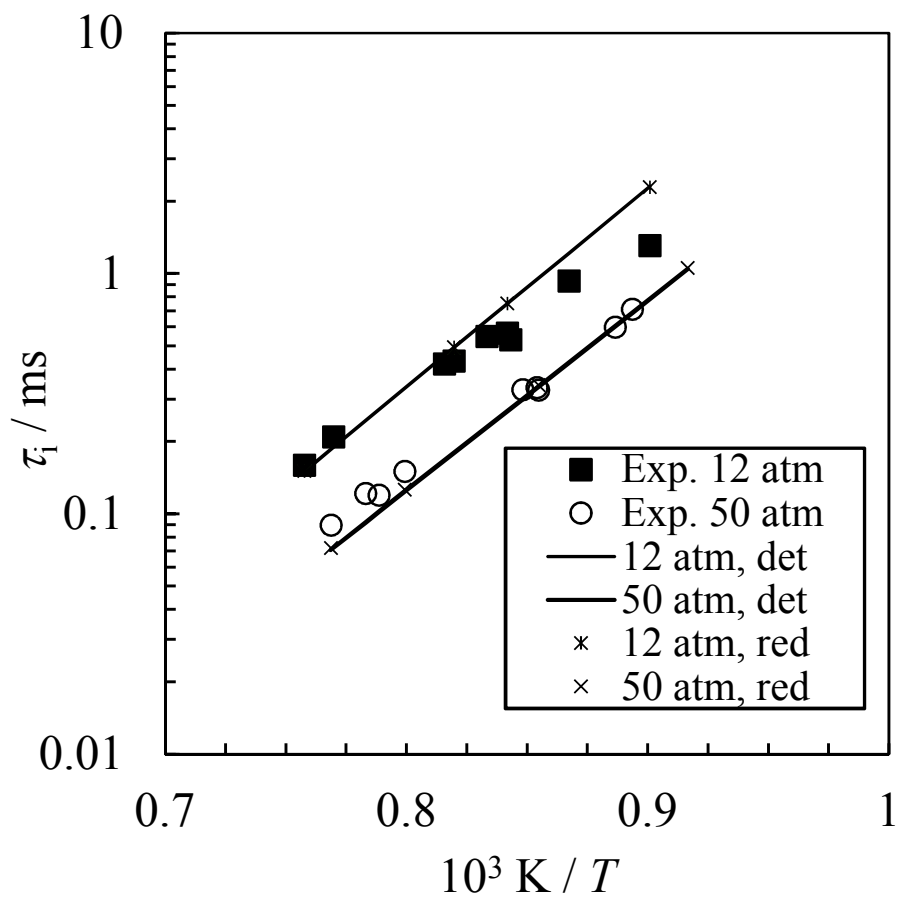

Fig. 3. Ignition time measurements for toluene/air mixtures by Shen et al. ${ }^{2}$ at 12 and 50 atm and $\phi=0.5$ and comparison to predictions of detailed (159 species) and reduced (123 species) kinetic mechanisms. Measured ignition times were scaled to 12 and $50 \mathrm{~atm}$, to account for slight deviations in reflected shock pressure, by $\tau_{\mathrm{i}} \propto p^{-0.50}$ as determined by regression analysis. 


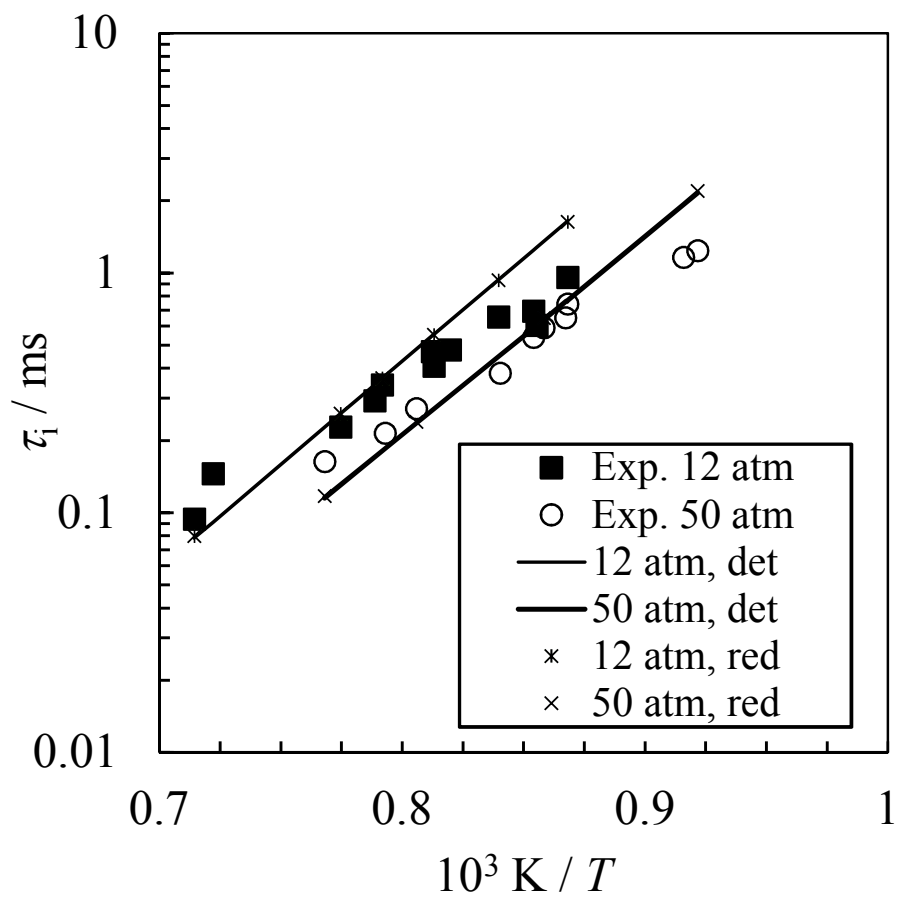

Fig. 4. Ignition time measurements for toluene/air mixtures by Shen et al. ${ }^{2}$ at 12 and 50 atm and $\phi=0.25$ and comparison to predictions of detailed (159 species) and reduced (123 species) kinetic mechanisms. Measured ignition times were scaled to 12 and $50 \mathrm{~atm}$, to account for slight deviations in reflected shock pressure, by $\tau_{\mathrm{i}} \propto p^{-0.23}$ as determined by regression analysis. 


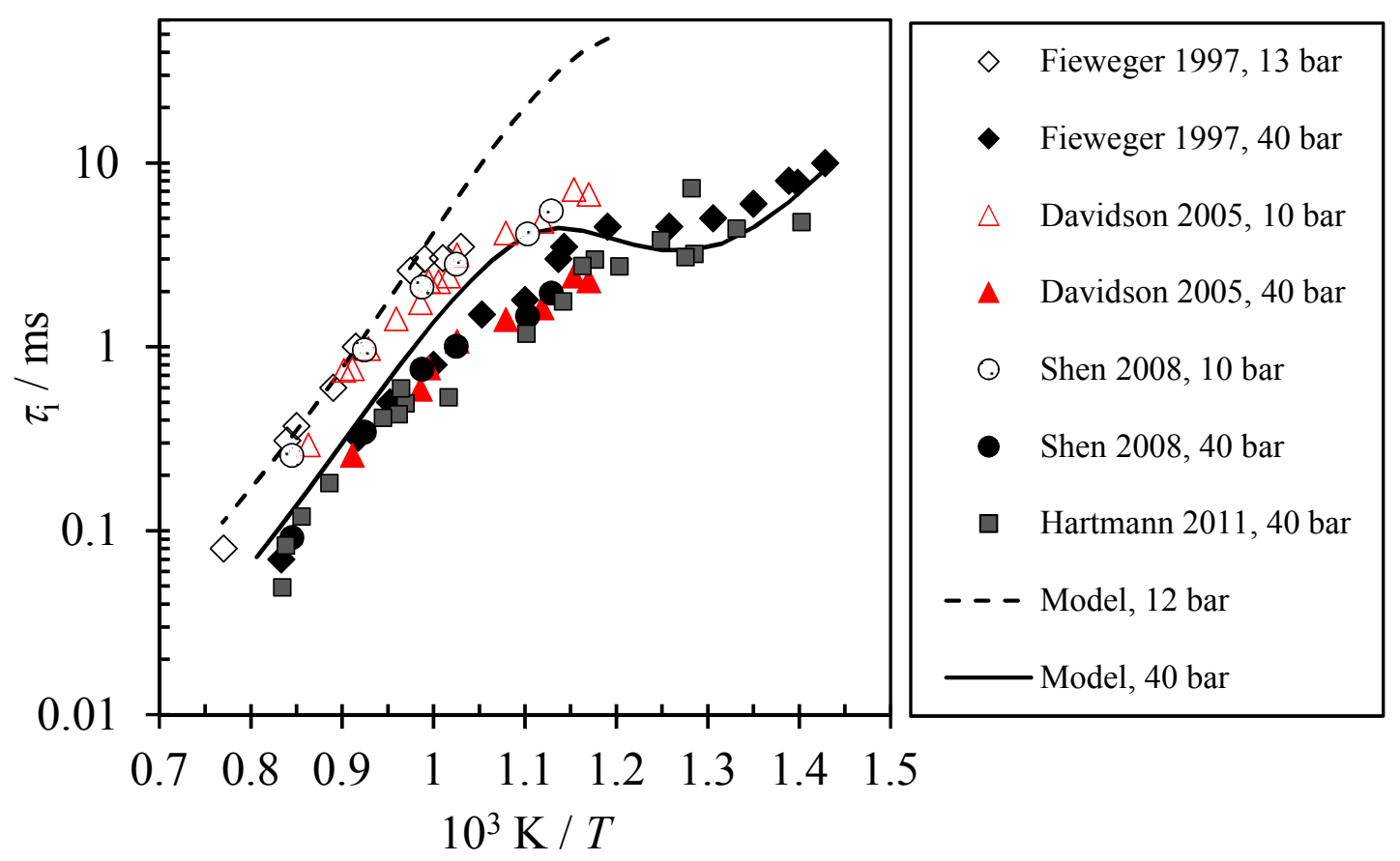

Fig. 5. Ignition time measurements (symbols) for iso-octane/air mixtures at $\phi=1.0$ and comparison to model predictions. ${ }^{3-6}$ For some data, ignition times were scaled to 10,12 and 40 bar, to account for slight deviations in reflected shock pressure, by $\tau_{\mathrm{i}} \propto p^{-0.75}[(3)]$ and $\tau_{\mathrm{i}} \propto p^{-0.79}[(5)]$ respectively. 


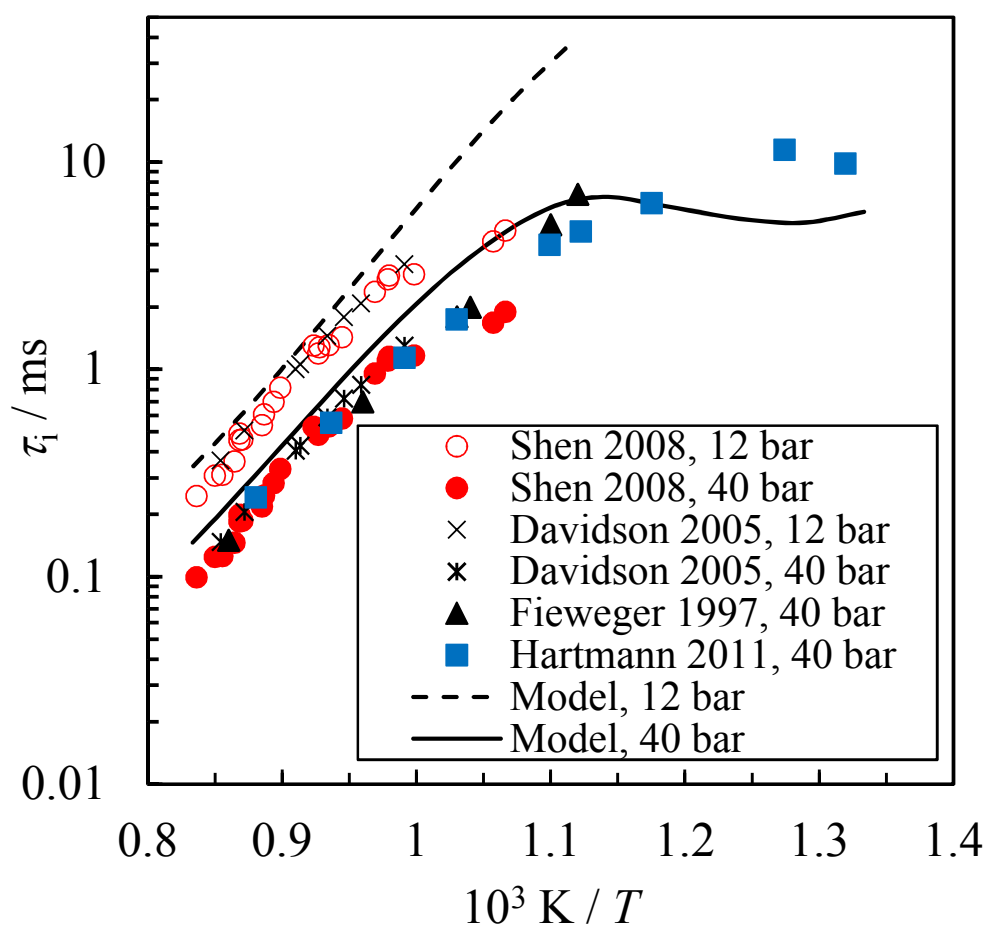

Fig. 6. Ignition time measurements (symbols) ${ }^{3-6}$ for iso-octane/air mixtures at 12 and 40 bar and $\phi=0.5$ and comparison to model predictions. Measured ignition times were for some data scaled to 12 and 40 bar, to account for slight deviations in reflected shock pressure, by $\tau_{\mathrm{i}} \propto p^{-0.75}$ as determined by regression analysis., 3 


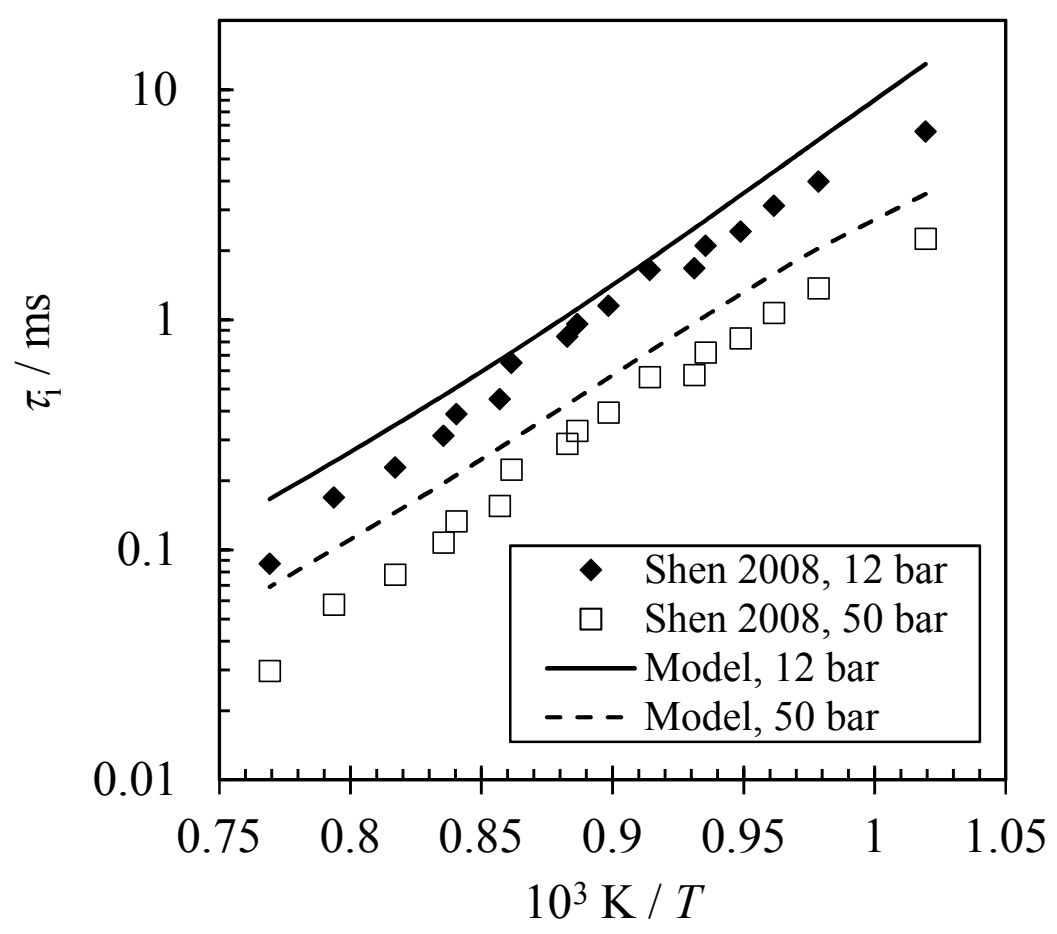

Fig. 7. Ignition time measurements for iso-octane/air mixtures ${ }^{3}$ at 12 and 50 bar and $\phi=0.25$ and comparison to model predictions. Measured ignition times were scaled to 12 and 50 bar, to account for slight deviations in reflected shock pressure, by $\tau_{\mathrm{i}} \propto p^{-0.75}$ as determined by regression analysis. 
N-heptane

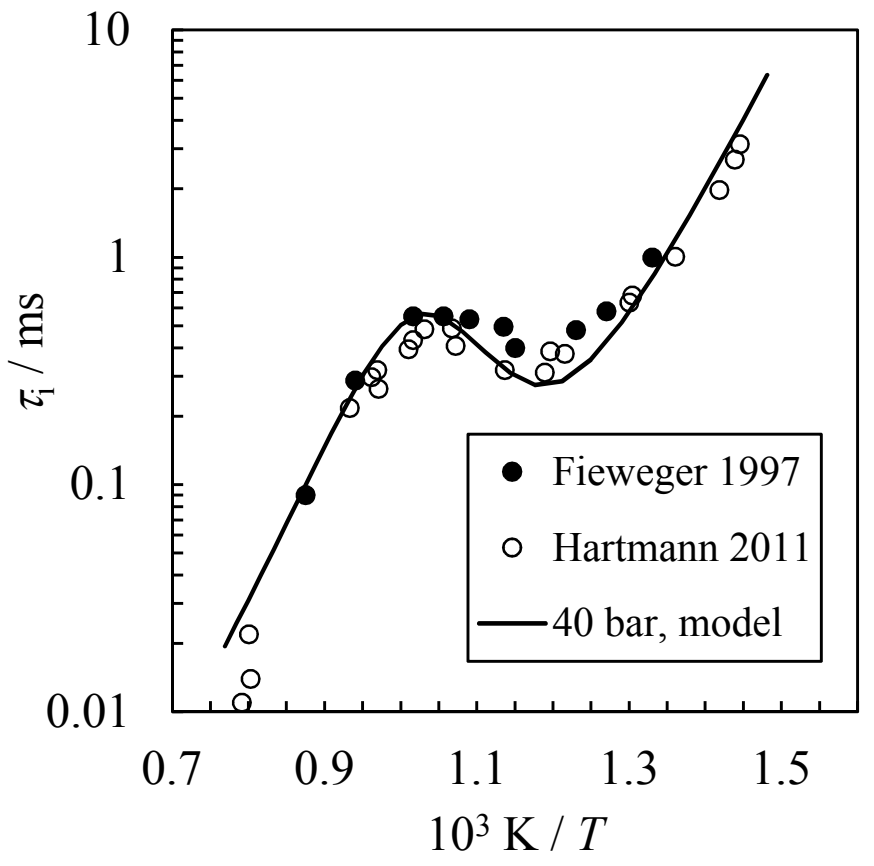

Fig. 8 Ignition time measurements for n-heptane/air mixtures at $40 \mathrm{bar}^{4,6}$ and $\phi=1.0$ and comparison to model predictions. 


\section{$\underline{\text { S2.2 Validation for binary fuel mixtures }}$}

Iso-octane/n-heptane (PRF)

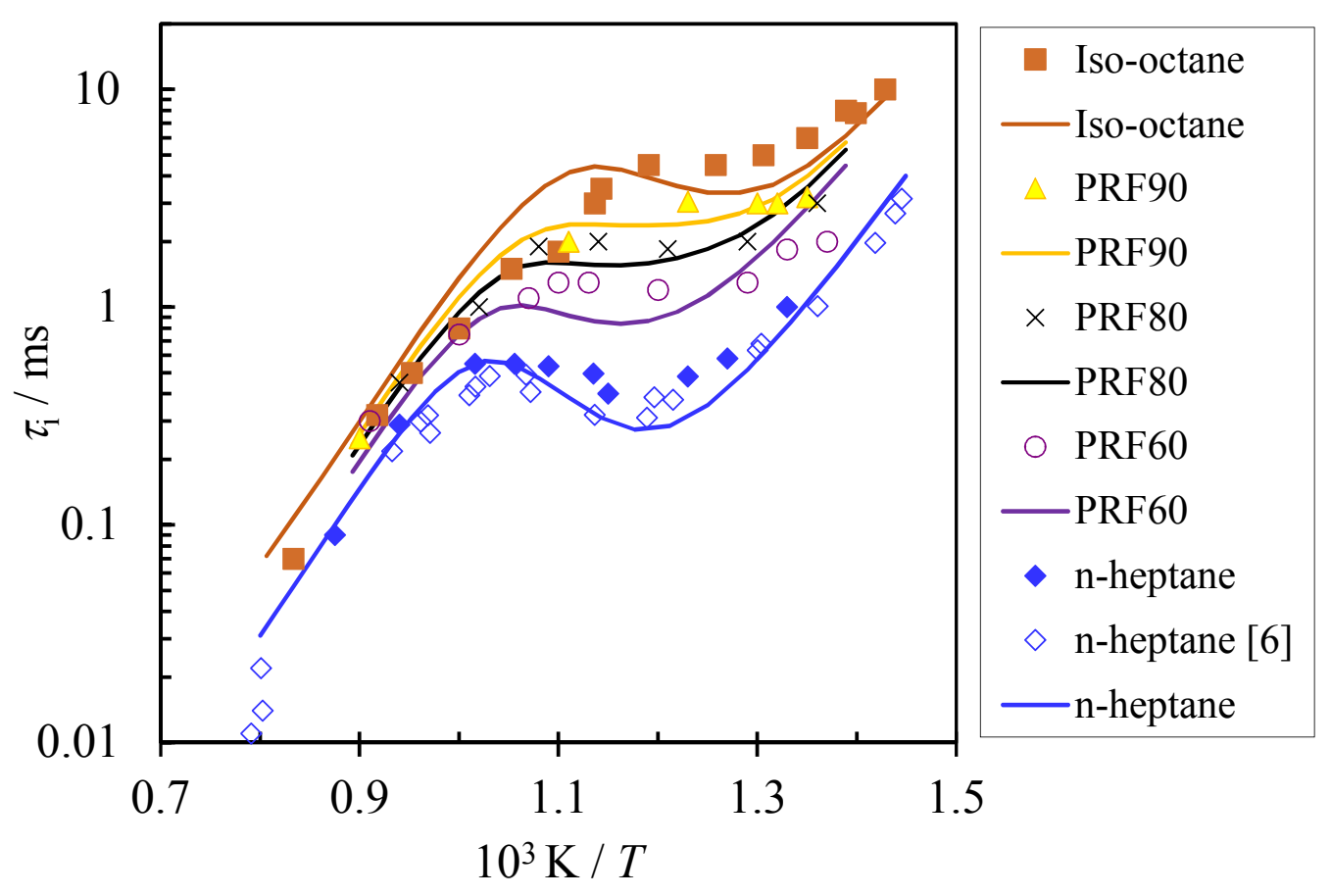

Fig. 9. Comparison of measured ${ }^{4,6}$ and simulated ignition delay for primary reference fuels in air for $p=40$ bar and $\phi=1.0$. Symbols - Experiments, Lines - Simulations with model in this work. 


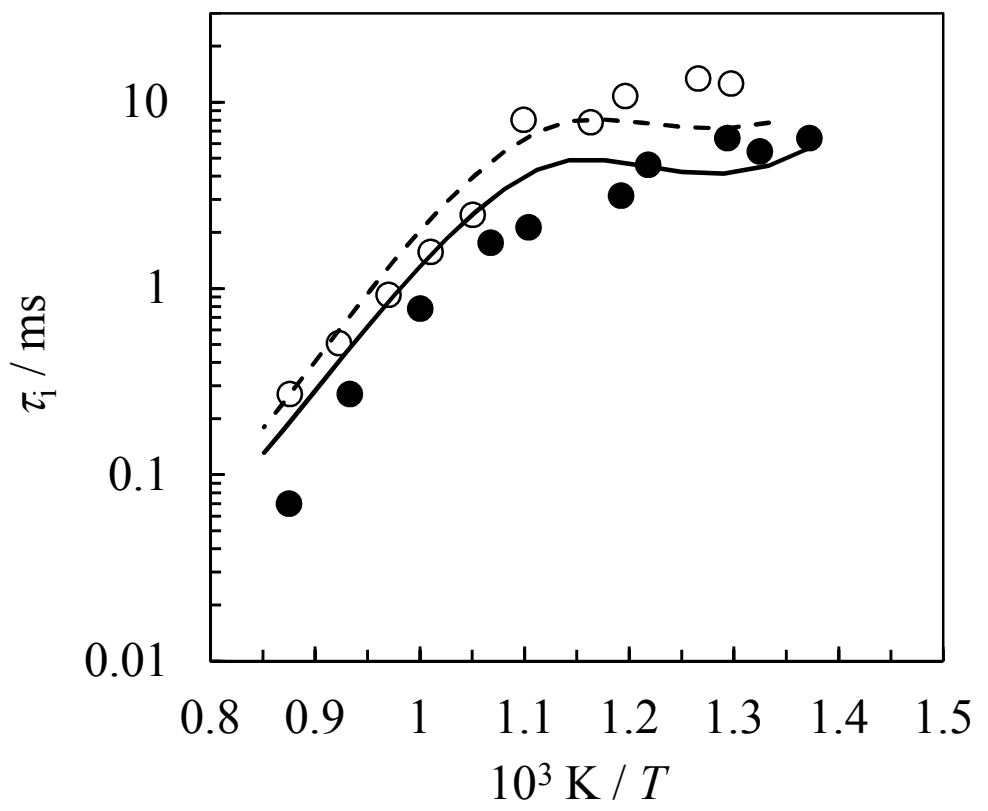

Fig. 10. Comparison of measured ${ }^{6}$ and simulated ignition delay for toluene/iso-octane (10/90 vol. \%) mixtures in air for $p=40$ bar. (•) Exp. $\phi=1.0,(-)$ Simulation $\phi=1.0,(\circ)$ Exp. $\phi=0.5$, $(--)$ Simulation $\phi=0.5$. 


\section{Toluene/n-heptane}

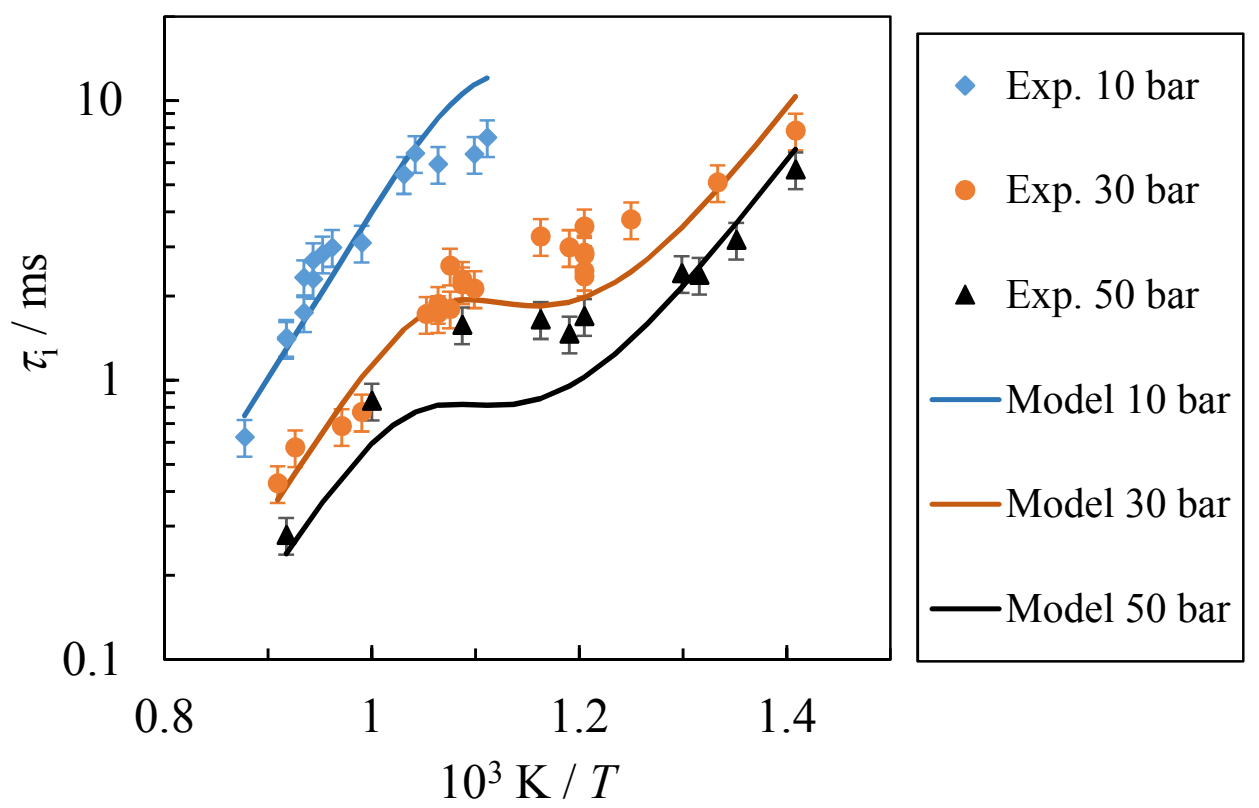

Fig. 11. Comparison of measured ${ }^{7}$ and simulated ignition delay for toluene/n-heptane (65/35 vol. $\%)$ mixtures for $\phi=1.0$. Measured ignition times were scaled to 10,30 and 50 bar by $\tau_{\mathrm{i}, \text { tol/hep }} \propto p^{-1.059}$.

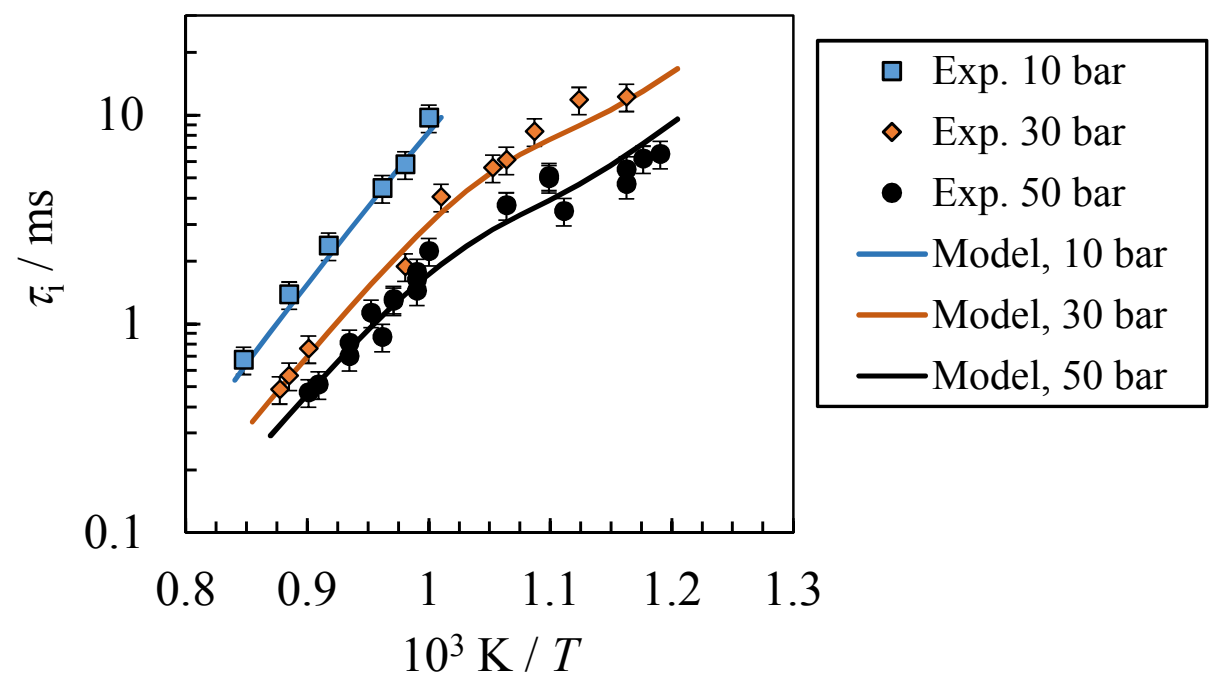

Fig. 12. Comparison of measured ${ }^{7}$ and simulated ignition delay for toluene/n-heptane (65/35 vol. $\%$ ) mixtures for $\phi=0.3$. Measured ignition times were scaled to 10,30 and 50 bar by $\tau_{\mathrm{i}, \text { tol } / \text { hep }} \propto p^{-0.883}$. 


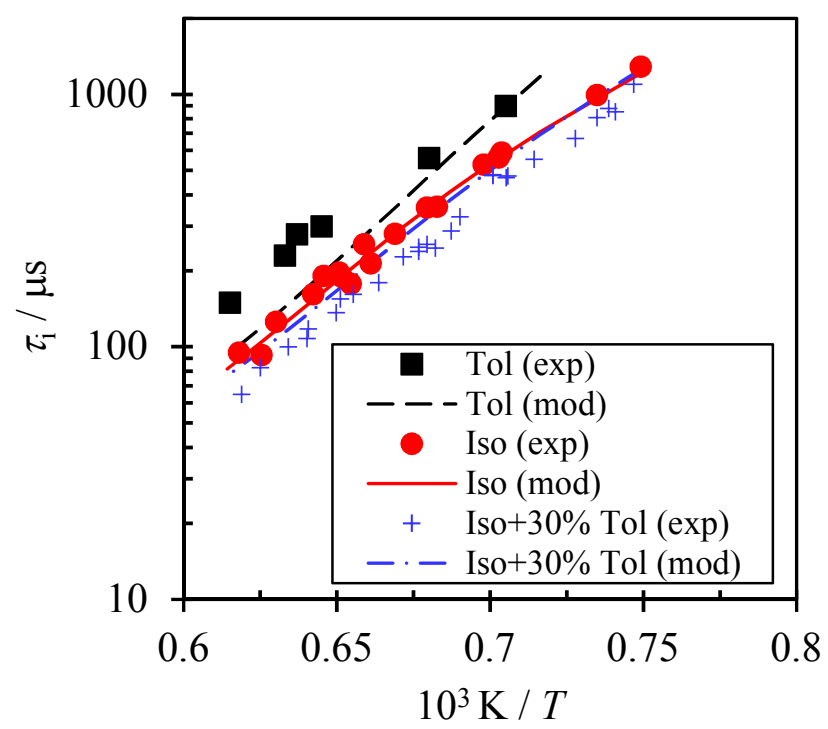

Fig. 13 Experimental ${ }^{8}$ ignition delay times behind reflected shock waves compared to simulations using the detailed model in this work (constant volume). Mole fraction fuel $=0.004, \phi=1.0$, balance $\mathrm{Ar}, p=2$ atm.

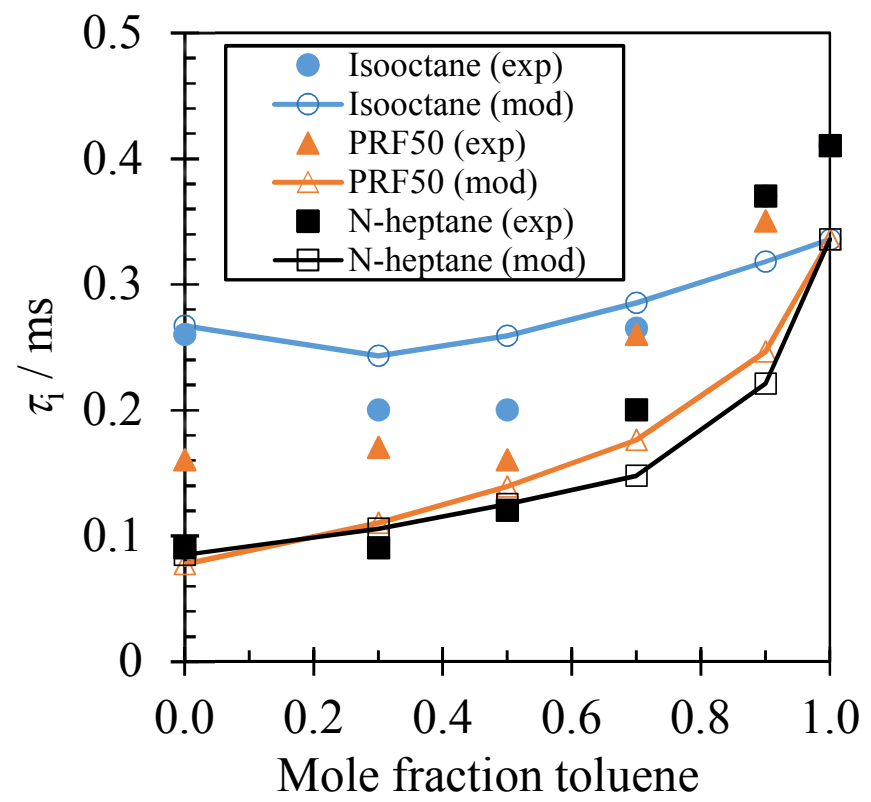

Fig. 14 Variation of experimental ${ }^{8}$ ignition delay times behind reflected shock waves with the addition of toluene to n-heptane, isooctane and isooctane $50 \% / \mathrm{n}$-heptane $50 \%$ compared to simulations using the detailed model in this work (constant volume). Mole fraction fuel $=0.004$, $\phi=1.0$, balance $\mathrm{Ar}, p=2 \mathrm{~atm}, T=1500 \mathrm{~K}$. 


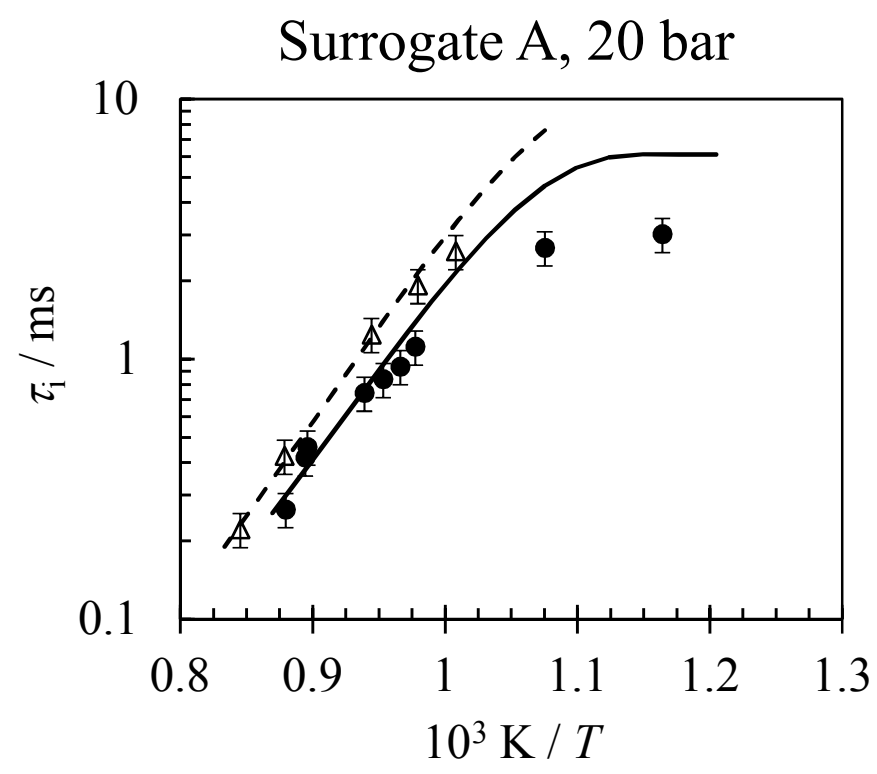

Fig. 15. Comparison of measured ${ }^{9}$ and simulated ignition delay for iso-octane/toluene/n-heptane $(63 / 20 / 17$ vol. \%) mixtures in air at $p=20$ bar. (๑) Exp. $\phi=1.0,(-)$ Simulation $\phi=1.0,(\triangle)$ Exp. $\phi=0.5,(--)$ Simulation $\phi=0.5$. Measured ignition times were scaled to 20 bar by $\tau_{\mathrm{i}, \propto} p^{-0.83}$.

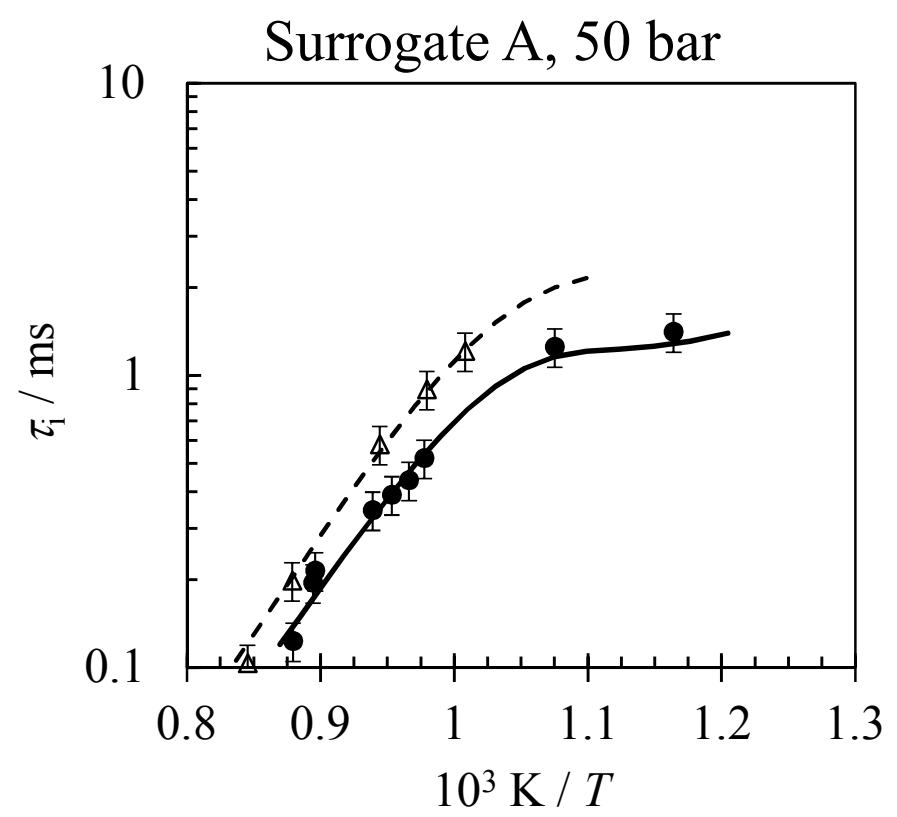

Fig. 16. Comparison of measured ${ }^{9}$ and simulated ignition delay for iso-octane/toluene/n-heptane $(63 / 20 / 17$ vol. \%) mixtures in air at $p=50$ bar. (๑) Exp. $\phi=1.0,(-)$ Simulation $\phi=1.0,(\triangle)$ Exp. $\phi=0.5,(--)$ Simulation $\phi=0.5$. Measured ignition times were scaled to 50 bar by $\tau_{\mathrm{i},} \propto p^{-0.83}$. 


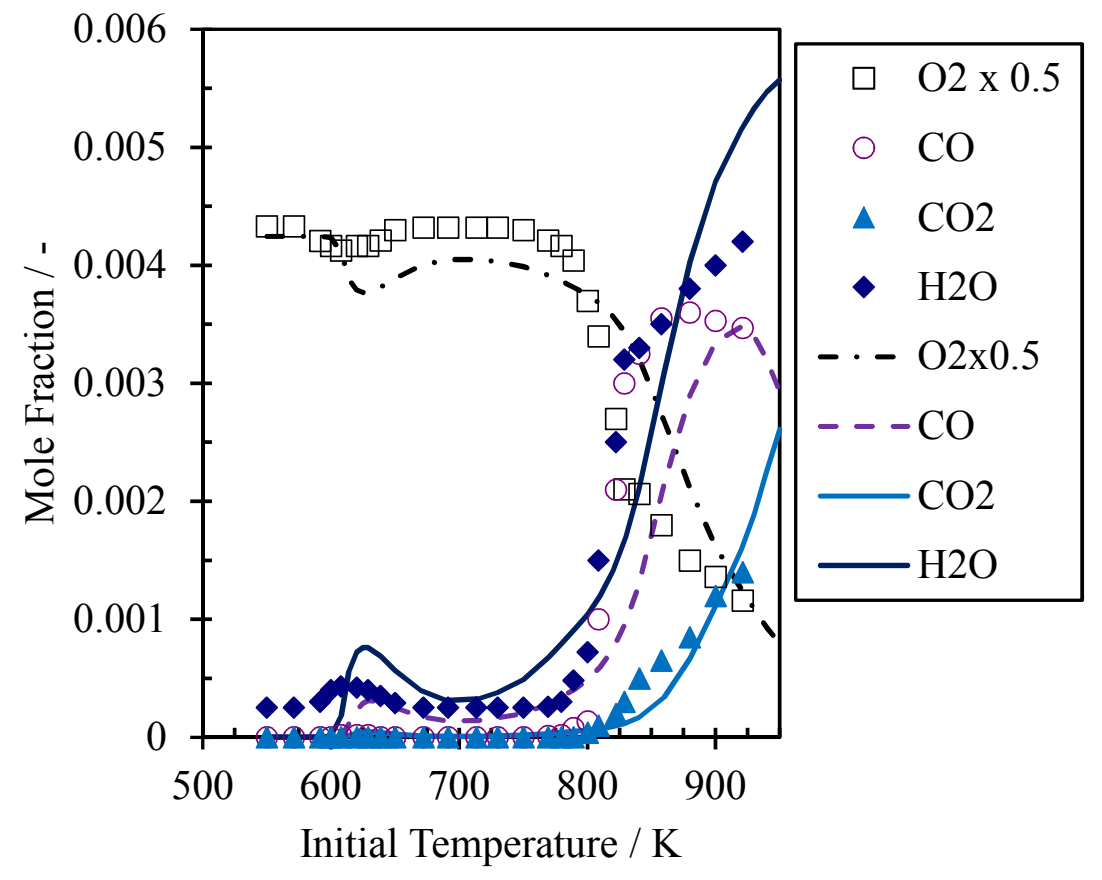

Fig. 17 Stable species profiles of a stoichiometric ternary mixture (18.23\% n-heptane, $66.42 \%$ isooctane, $15.35 \%$ toluene by liq. vol.)/air combustion in a flow reactor at $12.5 \mathrm{~atm}$ with a residence time of $1.8 \mathrm{~s}$ and $740 \mathrm{ppm}$ initial fuel fraction. Symbols denote the experimental measurements by Chaos et al. ${ }^{10}$ Lines show the numerical results for the model in this work. 


\section{$\underline{\text { S2.4 Combustion timing in HCCI }}$}

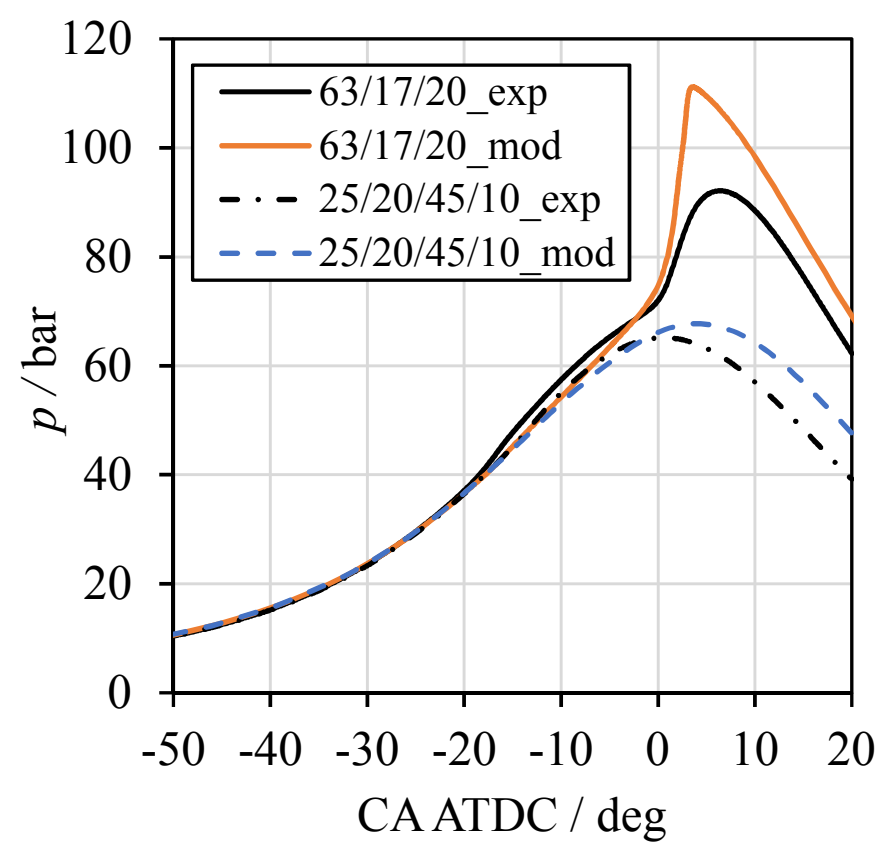

Fig. 18 Combustion timing in HCCI. Experiment by Andrae and Head ${ }^{11}$ with fuel compositions as iso-octane/n-heptane/toluene/DIB-1 by vol-\%. Conditions for simulations using a single zone model: $\phi=0.25, \mathrm{p}_{-99}=3.2 \mathrm{bar}, \mathrm{T}_{-99}=435 \mathrm{~K}, 1200 \mathrm{rpm}$. Convective heat loss modeled using a reduced compression ratio of 13.1 (geometrical compression ratio is 14.04). 


\section{$\underline{\text { S2.5 Laminar burning velocity }}$}

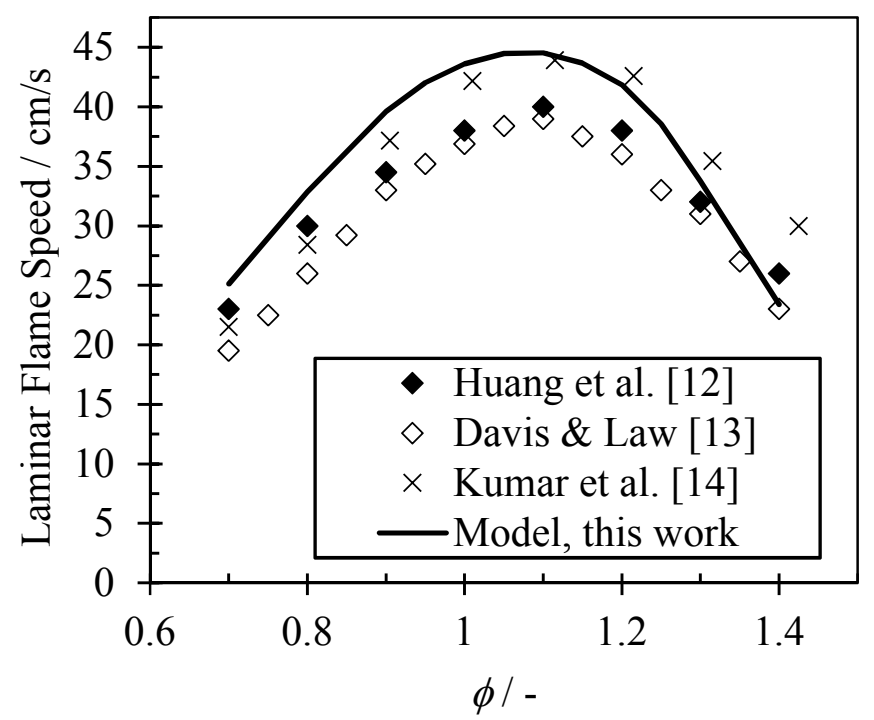

Fig. 19 Comparison of measured ${ }^{12-14}$ and modeled (reduced model) laminar burning velocities of atmospheric n-heptane/air flames at room temperature.

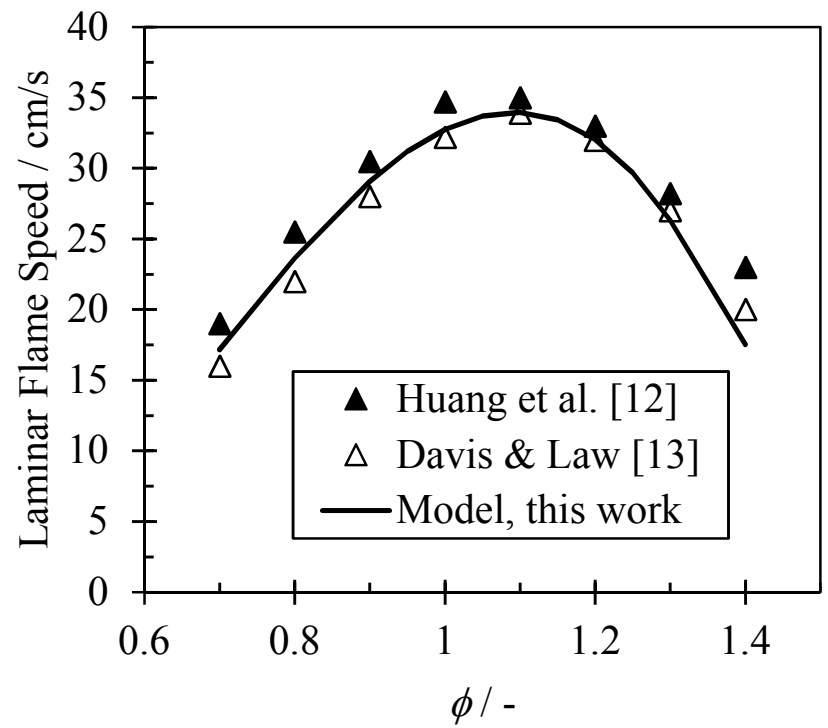

Fig. 20 Comparison of measured ${ }^{12,13}$ and modeled (reduced model) laminar burning velocities of atmospheric iso-octane/air flames at room temperature. 


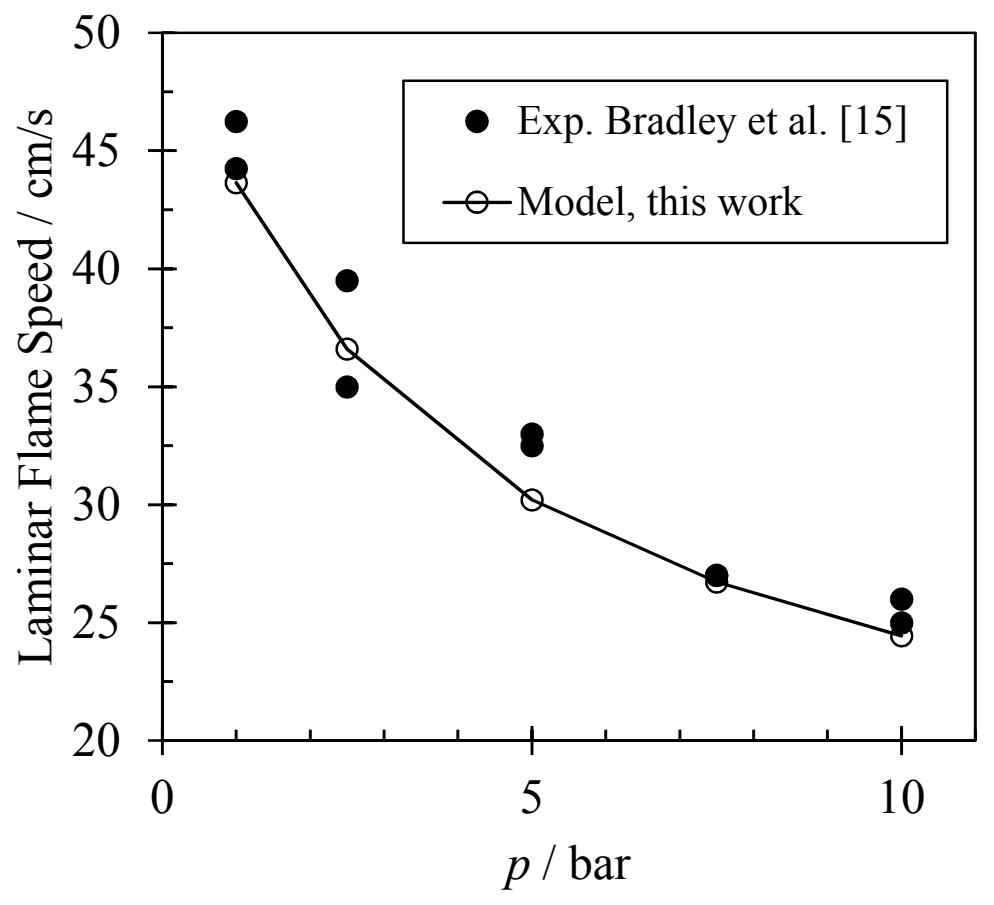

Fig. 21 Variation of laminar burning velocity with pressure for stoichiometric iso-octane-air mixtures. $T_{\mathrm{u}}=358 \mathrm{~K}$.

\section{$\underline{\text { S2.6 References }}$}

(1) Metcalfe, W. K.; Pitz, W. J.; Curran, H. J.; Simmie, J. M.; Westbrook C. K. Proc. Combust. Inst. 2007, 31, 377-384.

(2) Shen, H. P. S.; Vanderover, J.; Oehlschlaeger, M. A. Proc Combust Inst 2009, 32, 165-72.

Shen, H. P. S.; Vanderover, J.; Oehlschlaeger, M. A. Combust. Flame 2008, 155, $739-755$.

Fieweger, K.; Blumenthal, R.; Adomeit, G. Combust. Flame 1997, 109, 599-619.

Davidson, D. F.; Gauthier, B. M.; Hanson, R. K. Proc. Combust. Inst. 2005, 30, $1175-1182$.

Hartmann, M.; Gushterova, I.; Fikri, M.; Schulz, C.; Schieß1, R.; Maas, U. Combust. Flame 2011, 158, 172-178.

Herzler, J.; Fikri, M.; Hitzbleck, K.; Starke, R.; Schulz, C.; Roth, P.; Kalghatgi G. T. Combust. Flame 2007, 149, 25-31.

Sakai, Y.; Ozawa, H.; Ogura, T.; Miyoshi, A.; Koshi, M.; Pitz, W. J. SAE Tech. Pap. Ser. 2007, DOI: 10.4271/2007-01-4104 
(9) Gauthier, B. M.; Davidson, D. F.; Hanson, R. K. Combust. Flame 2004, 139, 300311.

(10) Chaos, M.; Zhao, Z.; Kazakov, A.; Gokularkrishnan, P.; Angioletti, M.; Dryer, F. L. A PRF+toluene surrogate fuel model for simulating gasoline. Kinetics Paper E26, Proceedings of the $5^{\text {th }}$ U. S. Combustion Meeting, 2007.

(11) Andrae, J. C. G.; Head, R. A. Combust. Flame 2009, 156, 842-51.

(12) Huang, Y.; Sung, C. J.; Eng, J. A. Combust. Flame 2004, 139, 239-251.

(13) Davis, S. G.; Law, C. K. Proc Combust Inst 1998, 27, 521-527.

(14) Kumar, K.; Freeh, J. E.; Sung, C. J.; Huang, Y. J. Prop. Power 2007, 23, 428-436.

(15) Bradley, D.; Hicks, R. A.; Lawes, M.; Sheppard, C. G. W.; Wooley, R. Combust. Flame 1998, 115, 126-144. 